\title{
MEDIDAS ALTERNATIVAS DE INFLACIÓN
}

\author{
Calixto Mateos y Alejandro Gaytán*
}

cmateos@ banxico.org.mx y agaytan@ banxico.org.mx

\author{
Julio de 1998 \\ D ocumento de Investigación No. 9802 \\ Dirección General de Investigación Económica \\ BANCO DE MÉXICO
}

\footnotetext{
* Los autores son Subgerente de Programación Macrofinanciera y Economista, respectivamente en la Dirección de Estudios Económicos. Se agradece la excelente ayuda de investigación de Mercedes Sánchez Reyes Retana así como los comentarios de Stephen Cecchetti, Agustín Carstens, Abraham Vela, Alejandro Werner y Moisés Schwartz. Las opiniones contenidas en el documento corresponden exclusivamente a los autores y no representan necesariamente el punto de vista del Banco de México.
} 


\title{
MEDIDAS ALTERNATIVAS DE INFLACIÓN
}

\author{
Calixto Mateos y Alejandro Gaytán \\ cmateos@ banxico.org.mx y agaytan@ banxico.org.mx
}

Julio de 1998

D ocumento de Investigación No. 9802

\begin{abstract}
RESUMEN
El crecimiento del índice de precios al consumidor es el indicador más utilizado para medir la inflación. Sin embargo, dicho indicador puede reflejar variaciones en precios relativos o algunas otras perturbaciones cuyo origen radique en consideraciones ajenas a la política monetaria. En este documento se reseñan y se aplican al caso de México las metodologías para calcular una serie de medidas que han sido desarrolladas con el objetivo de depurar al índice de precios al consumidor de las variaciones temporales o de precios relativos de manera que el indicador así obtenido sea una medida de inflación que capture únicamente el crecimiento sostenido en el nivel general de precios. Cada una de estas medidas provee información sobre algún aspecto del proceso inflacionario, por lo que resulta importante que el seguimiento de estas medidas se incorpore dentro del análisis de la inflación en México.
\end{abstract}




\section{Contenido}

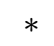

I. INTRODUCCIÓN ……………………………………………………………………………...1

II. DESCRIPCIÓN DE D IVERSO S INDICADORES DE INFLACIÓN ………………………….... 2

III. INFLACIÓN SUBYACENTE ..................................................................................................... 4

IV. NÚCLEO INFLACIONARIO ……………………………………………………………....

V. MEDIDAS DE INFLACIÓN CON ESTIMADORES DE INFLUENCIA LIMITADA ........... 12

VI. INFLACIÓN LATENTE ............................................................................................................. 16

VII. INFLACIÓN PERMANENTE ................................................................................................. 21

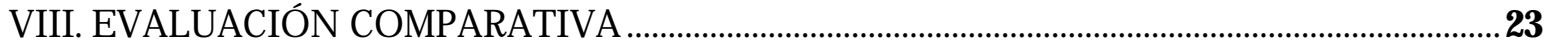

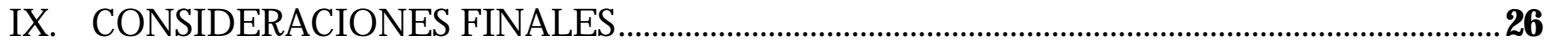

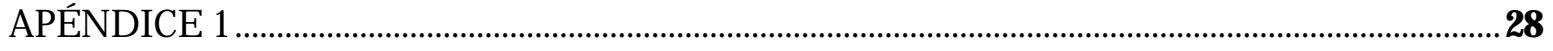

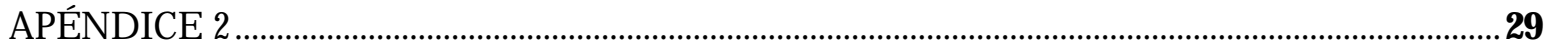

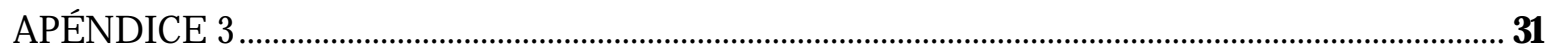

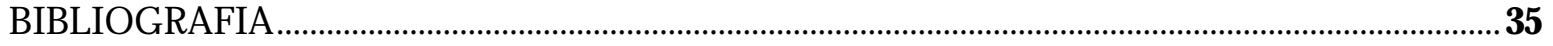




\section{MEDIDAS ALTERNATIVAS DE INFLACIÓN}

\section{INTRODUCCIÓN}

Uno de los fenómenos que tradicionalmente ha recibido la mayor atención por parte de los economistas es la inflación. La inflación posiblemente no estaría entre los principales tópicos económicos si fuera únicamente un fenómeno de crecimiento de las magnitudes nominales, sin un efecto sobre el sector real. Sin embargo, existe una extensa literatura que documenta tanto teórica como empíricamente los múltiples y variados efectos adversos de la inflación sobre la asignación de recursos, la distribución del ingreso, el desarrollo económico y, por ende, sobre el bienestar de la población. ${ }^{1}$

La inflación se define teóricamente como el crecimiento sostenido en el nivel general de los precios. Asimismo, es generalmente aceptado que en el largo plazo la inflación es un fenómeno puramente monetario. Por este motivo, el estudio de la inflación es de particular relevancia para los bancos centrales, especialmente para aquéllos cuyo mandato principal es la estabilidad del poder adquisitivo de la moneda.

En la práctica, el indicador más utilizado de inflación es el crecimiento del índice de precios al consumidor (IPC), sin embargo, este indicador no necesariamente refleja de forma adecuada el fenómeno inflacionario. El IPC está diseñado para medir el costo de la vida para un consumidor típico. Por construcción, este índice considera a un subconjunto de los precios de la economía, por lo que no es un índice diseñado para medir el cambio en el nivel general de los precios. Si bien es de esperarse que los precios de los bienes que conforman la canasta del IPC varíen simultáneamente con el nivel general de los precios, también es posible que lo hagan como resultado de variaciones en precios relativos $\mathrm{u}$ otras perturbaciones cuyo origen radique en consideraciones ajenas a la instrumentación de la política monetaria, tales como las asociadas con las fluctuaciones estacionales en precios agrícolas, las variaciones en los términos de intercambio, las modificaciones en los impuestos indirectos, los ajustes a precios y tarifas públicos y al salario mínimo. ${ }^{2}$

En consecuencia, modificaciones del índice de precios al consumidor que se originen únicamente por variaciones en precios relativos pueden conducir a una interpretación errónea del proceso inflacionario. Más aún, si las autoridades monetarias reaccionaran ante estas variaciones en precios relativos, ello podría imponer costos muy severos sobre el sector real de la economía. De ahí que, desde la perspectiva de la autoridad monetaria, sea importante contar con un indicador que refleje el crecimiento sostenido de los precios y no las fluctuaciones debidas a cambios en precios relativos. Por supuesto, este indicador será de gran relevancia para aquellos bancos centrales que instrumentan su política monetaria vía un esquema de metas de inflación. ${ }^{3}$

No obstante lo anterior, el IPC es en la actualidad la medida más conocida y oportuna que recaba información sobre un número importante de precios en la economía, de manera que si se le depurara

\footnotetext{
${ }^{1}$ Véase, por ejemplo: De Gregorio (1992) y (1993), Fischer (1993), Pindyck y Solimano (1993), Sbordone y Kuttner (1994), Smyth (1994), y Bruno y Easterly (1995).

${ }^{2}$ De acuerdo con la teoría clásica, los precios relativos están determinados por factores reales mientras que la oferta monetaria determina el nivel general de los precios. De manera que, para un nivel de dinero en la economía, cambios en precios relativos implicarían el aumento de algunos precios y la disminución de otros y, por tanto, el índice de precios no se alteraría necesariamente. No obstante lo anterior, Ball y Mankiw (1995) demuestran que en el corto plazo los costos de ajustar los precios, "costos de menú", producen rigideces en el mecanismo de ajuste de precios, lo que genera cambios en los precios relativos que son capturados por el índice de precios al consumidor.

${ }^{3}$ Una descripción del esquema de metas de inflación se encuentra en Mateos y Schwartz (1997).
} 
de las variaciones en precios relativos o de las perturbaciones exógenas, se podría tener un indicador de inflación que corresponda más cabalmente con su definición teórica. En este espíritu y como resultado de diversas metodologías han surgido medidas alternativas que buscan, mediante el uso de la información del IPC, medir el crecimiento de los precios que refleje más cercanamente al aumento sostenido en el nivel general de precios. ${ }^{4}$

En este documento se reseña la discusión que en los últimos años ha surgido a este respecto y se presentan las metodologías para el cálculo de diversas medidas que son utilizadas para determinar a la inflación. Aunque en la literatura a cada una de estas medidas se le ha llamado indistintamente inflación subyacente, núcleo inflacionario o inflación núcleo, en este trabajo se seguirá la nomenclatura propuesta por Alvarez y Matea (1997). En particular, se consideran los conceptos de inflación subyacente, núcleo inflacionario, medidas de inflación con estimadores de influencia limitada, inflación latente e inflación permanente.

El documento se organiza de la siguiente manera, en la segunda sección se hace una breve reseña de las consideraciones que han motivado este tipo de medidas. En las secciones subsecuentes se describe cada una de ellas y se presenta su estimación para México. En la octava sección se realiza una evaluación comparativa y en la última sección se presentan algunas consideraciones finales.

\section{DESCRIPCIÓN DE DIVERSOS IN DICADORES DE INFLACIÓN}

D esde hace varios años a los bancos centrales de diversos países les ha preocupado el efecto que sobre el IPC producen los precios volátiles y los pagos de intereses, ya que dicho efecto puede dar lugar a interpretaciones erróneas del proceso inflacionario que afecten la conducción de la política monetaria. Los cambios transitorios en los precios al consumidor debidos a efectos estacionales, a precios con alta volatilidad (como la energía), a cambios en precios administrados o a cambios en impuestos indirectos no reflejan las presiones inflacionarias subyacentes. Por ello, las autoridades monetarias de Francia, Italia, el Reino Unido, Canadá, Australia, Austria, Dinamarca, Holanda y Suecia, han argumentado que para efectos de la política monetaria lo relevante son la variaciones de los precios que perduran en el largo plazo, es decir, la tendencia del crecimiento de los precios.

Una solución propuesta a este problema ha sido obtener directamente la tendencia de la serie formada por el crecimiento porcentual del IPC. La tendencia de una serie de tiempo se puede obtener mediante la utilización de medias móviles, modelos econométricos ARIMA $^{5}$ y filtros estadísticos, entre otros. Sin embargo, cada una de estas técnicas presenta algún inconveniente. Por ejemplo, el uso de medias móviles tiene la ventaja de que la tendencia que se obtiene es invariante a nueva información, pero presentan la desventaja de que sólo es posible conocer dicha tendencia con un rezago considerable. Por otra parte, las técnicas de modelos ARIMA o filtros estadísticos permiten obtener una medida tendencial contemporánea, pero tienen el inconveniente de que dependen del tamaño de muestra y que el resultado tendencial obtenido para el periodo corriente puede cambiar con la incorporación de nuevas observaciones. Alvarez y Matea (1997) clasifican a la medida de inflación tendencial como "inflaciónsubjacente".

Otra solución propuesta para depurar al IPC de los cambios transitorios en precios relativos ha sido el construir una medida de inflación que explícitamente excluye los precios de los bienes y servicios considerados más volátiles; tales como los precios de los alimentos, la energía, los impuestos indirectos, y los pagos de intereses entre otros; a dicha medida se le ha llamado indistintamente núcleo

\footnotetext{
${ }^{4}$ Es obvio que existen diversas consideraciones metodológicas sobre la construcción del IPC que podrían ser incorporadas para que éste se convirtiera en un mejor indicador de la inflación o del costo de la vida (Armknecht (1996) presenta estas consideraciones para el caso de los Estados Unidos). Este trabajo no sigue esta línea de investigación.

${ }^{5}$ ARIMA son las siglas en inglés para un modelo integrado autorregresivo de medias móviles.
} 
inflacionario (cre inflation) o inflación subyacente (undetying inflation). Siguiendo a Alvarez y Matea (1997) en este trabajo se designará a esta medida como "núdœ inflacionano". Este tipo de medidas tienen el inconveniente de que al computar un nuevo índice que elimina a prior los precios de algunos bienes y servicios se corre el riesgo de desechar información importante del proceso inflacionario. Adicionalmente, no existe una metodología específica para la determinación de los elementos a excluir.

Recientemente se han realizado algunos esfuerzos para construir una medida de inflación más cercana a su definición teórica. En particular, Bryan y Cecchetti (1993) buscan extraer del IPC una medida de la tendencia inflacionaria que sea oportuna y que no se modifique con la incorporación de nuevas observaciones. Para ello utilizan "estimadores deinfluenaa limitada" sobre la distribución de los cambios en los precios que componen al IPC. El procedimiento también contempla la construcción de un nuevo índice que excluye algunos bienes y servicios, sin embargo, permite eliminar los precios de distintos bienes y servicios en cada periodo y propone un criterio para determinar cuáles elementos eliminar. Bryan y Cecchetti demuestran que, para el caso de EUA, estos nuevos índices de precios presentan una relación más estrecha con los agregados monetarios que aquéllos que a prioi excluyen algunos elementos.

En otra línea de investigación Quah y Vahey (1995) argumentan que si bien el uso de estimadores de influencia limitada puede producir una medida de inflación menos distorsionada que el cambio porcentual del IPC, en algunos casos puede no ser así y que además dicha técnica, al excluir los precios de algunos bienes y servicios, continúa desechando información importante sobre el proceso inflacionario.

Quah y Vahey proponen un enfoque diferente para estimar la inflación. En lugar de calcular un nuevo índice que elimina algunos bienes y servicios, definen a la inflación como aquella parte del crecimiento del IPC que no tiene un impacto del mediano al largo plazo sobre la actividad económica real. Así, esta medida de inflación en el largo plazo no tendrá relación con las variables reales, por lo que será congruente con la dicotomía clásica, y por ende, con una interpretación de los movimientos de los precios y de la producción congruente con una curva de Phillips vertical en el largo plazo. ${ }^{6}$

Para estimar esta medida, es necesario incorporar, además de toda la información contenida en el IPC, la información sobre el nivel de actividad económica y utilizar la metodología de VAR estructural propuesta por Blanchard y Quah (1989). El inconveniente de esta metodología es que es esencialmente paramétrica y que los resultados de toda la muestra pueden variar si los parámetros estimados cambian al incorporar nuevas observaciones. A esta medida se le denomina "inflación latente'.

Alvarez y Sebastián (1995) mediante un cambio en la metodología de Quah y Vahey obtienen otra medida de inflación a la que se denomina "inflacón pemanente". La inflación permanente captura los cambios del crecimiento del IPC que perduran en el tiempo. Por este motivo, la inflación permanente es un buen indicador del cambio sostenido en el nivel general de precios, mostrando el movimiento tendencial de la inflación sin eliminar información de los precios que componen al IPC.

Cada una de las metodologías mencionadas permite obtener una medida representativa que supera en parte las desventajas del cambio porcentual del IPC como indicador inflacionario. A pesar de que ninguna de estas medidas queda libre de algún inconveniente, cada una provee información de algún aspecto del proceso inflacionario, por lo que resulta importante que el seguimiento de estas medidas se incorpore dentro del análisis de este fenómeno económico. En las siguientes secciones se describe con

\footnotetext{
${ }^{6}$ Las observaciones originales de Phillips (1958) se refirieron a la relación entre la tasa de cambio de los salarios y la tasa de desempleo.
} 
detalle y se realiza un cálculo para México de cinco medidas que corresponden a las cinco metodologías reseñadas.

\section{INFLACIÓN SUBYACENTE}

La "inflación subyacente" es la que resulta de obtener la tendencia de la serie formada por los cambios porcentuales mensuales del índice de precios al consumidor. La tendencia de una serie de tiempo constituye el componente libre de movimientos estacionales e irregulares de la misma, por lo que refleja una evolución más estable de la serie.

Existen diversas metodologías para extraer la tendencia de una serie de tiempo, entre estas metodologías se encuentran: el cálculo de medias móviles centradas y la utilización de técnicas estadísticas de extracción de señales con el fin de descomponer las variables en la suma de componentes no observables, a saber, el componente estacional, el componente irregular, el componente cíclico y, por supuesto, el componente tendencial. Entre estas técnicas se pueden citar al uso de modelos ARIMA o al uso de filtros estadísticos (Beveridge-Nelson, Kalman, entre otros).

Cada una de estas metodologías presenta algunas ventajas e inconvenientes. Por ejemplo, el uso de medias móviles centradas tiene la gran ventaja de su sencillez y de que la tendencia que se obtiene es invariante a nueva información, pero tiene el inconveniente de que sólo es posible conocer dicha tendencia con un rezago considerable. Por otra parte, las técnicas de modelos ARIMA o filtros estadísticos permiten obtener una medida tendencial contemporánea; sin embargo, tienen el inconveniente de que dependen del tamaño de muestra y que, por ser en general procesos paramétricos, la tendencia obtenida durante el periodo puede cambiar con la incorporación de nuevas observaciones.

Tanto el cálculo de medias móviles centradas, como los modelos ARIMA o los filtros estadísticos requieren de que se realicen ciertos supuestos. En el caso de la media móvil es necesario definir la dimensión temporal y la posición de la "ventana"; es decir, del número de observaciones previas y posteriores que se incluirán en el promedio. En los modelos ARIMA es necesario seleccionar el orden de rezagos de los procesos. Los filtros estadísticos requieren diferentes supuestos, por ejemplo, un filtro de Kalman requiere de una hipótesis sobre la forma funcional del proceso estocástico subyacente. Desafortunadamente, la teoría económica no ofrece ninguna predicción sobre estos supuestos, de manera que éstos quedan a criterio de cada investigador.

En esta sección se presentan para México dos cálculos de la inflación subyacente, uno basado en el cómputo de la media móvil centrada y el otro que resulta del componente tendencial de un modelo ARIMA, ambos sobre el crecimiento mensual del índice nacional de precios al consumidor (INPC).

La media móvil centrada para un mes en particular es el promedio de observaciones anteriores y posteriores a ese periodo en la serie original ${ }^{7}$, por lo que en general las medias móviles centradas sólo se encuentran disponibles con rezagos considerables. Este inconveniente con relación a las otras metodologías para obtener la tendencia, puede ser superado utilizando un pronóstico de la serie original para el número de periodos necesario para calcular la media móvil centrada para el periodo corriente. Sin embargo, este cómputo dependerá de la precisión del pronóstico y seguramente variará con la incorporación de nuevas observaciones por lo que la metodología de medias móviles centradas, así utilizada, presenta el mismo inconveniente que los modelos ARIMA o los filtros estadísticos.

${ }^{7}$ Cabe mencionar que el "centrado" no requiere necesariamente simetría en el número de observaciones anteriores y posteriores. 
Para determinar el tamaño y la posición de la "ventana" necesarios para calcular la inflación subyacente con la media móvil centrada para México, se siguió el criterio de que los máximos y mínimos de la medida subyacente coincidieran con los del crecimiento mensual del INPC. Así, después de probar distintas especificaciones, se concluyó que, para un mes en particular, la media móvil de 12 meses que incluye 2 observaciones anteriores y 9 posteriores a dicho mes representa adecuadamente la tendencia del crecimiento de los precios, es decir, la media móvil centrada en marzo de un año es el promedio de enero a diciembre de ese año. De manera que, este procedimiento de centrado requiere, para obtener el nivel corriente de la tendencia, del pronóstico de nueve observaciones futuras del INPC.

Los modelos ARIMA son el método univariado más flexible para modelar series de tiempo no estacionarias. ${ }^{8}$ Estos modelos permiten incorporar patrones de dependencia de la variable con respecto a sus rezagos - el elemento autorregresivo (AR)-- y con respecto a los valores presentes y pasados del término de error -el elemento promedio móvil (MA). Si la serie no es estacionaria, es necesario determinar su orden de integración (I) para que los procesos estadísticos autorregresivos y de promedios móviles sean válidos. ${ }^{9}$ El componente tendencial de un modelo ARIMA representa la evolución de largo plazo de la serie. Dicha tendencia tiene la ventaja de que incorpora los cambios en la media y la varianza de la serie original.

Para el caso de México, se obtuvo la tendencia del crecimiento mensual del INPC utilizando los programas TRAMO y SEATS desarrollados y utilizados por el Banco de España para el análisis de series de tiempo. ${ }^{10} \quad$ La selección del modelo ARIMA se basó en la condición estándar de que los residuales del modelo se comportaran como ruido blanco. El modelo ajustado se describe como ARIMA (pd,q)(lop,bd,bo), donde $\mathrm{p}$ representa el número de rezagos del componente autorregresivo regular, dindica el orden de integración requerido para que la serie regular sea estacionaria, y q es el orden de promedios móviles regulares. De igual manera, bpes el número de rezagos del componente autorregresivo estacional, bd es el orden de integración de la serie estacional y bq son los promedios móviles estacionales. El modelo ajustado correspondiente al crecimiento mensual del INPC en México fue un ARIMA $(0,1,1)(0,1,1){ }^{11}$

En la gráfica 1 se presentan estas dos medidas así como al crecimiento mensual del INPC. Como ahí se observa, las dos metodologías dan como resultado series de tendencia que, en general, reflejan la misma dirección de los cambios en el nivel de precios.

\footnotetext{
${ }^{8}$ Una variable (yt) es estacionaria cuando su media es constante y su varianza no crece en el tiempo. Formalmente, se dice

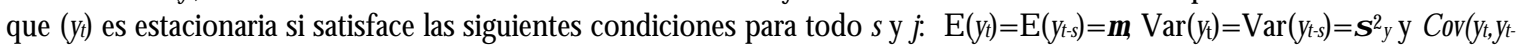

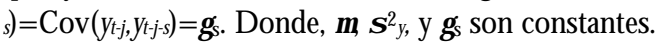

${ }^{9}$ Se dice que una serie (y) está integrada en orden cero $\mathrm{I}(0)$ cuando es estacionaria. En general, se dice que una serie está integrada con orden I(q) cuando su q-ésima diferencia es estacionaria.

${ }^{10}$ TRAMO y SEATS son los acrónimos de "Time Series Regression with ARIMA Noise, Missing Observations and Outliers" y "Signal Extraction in ARIMA Time Series", respectivamente.

${ }^{11}$ Para una descripción de la metodología del modelo ARIMA véase Maravall (1989), Espasa y Cancelo (1993), y Matea y Regil (1994). Para una descripción del uso de TRAMO y SEATS para estimar la tendencia del crecimiento del INPC en México véase Sánchez (1998).
} 


\section{Gráfica 1 \\ INFLACIÓN SUBYACENTE}

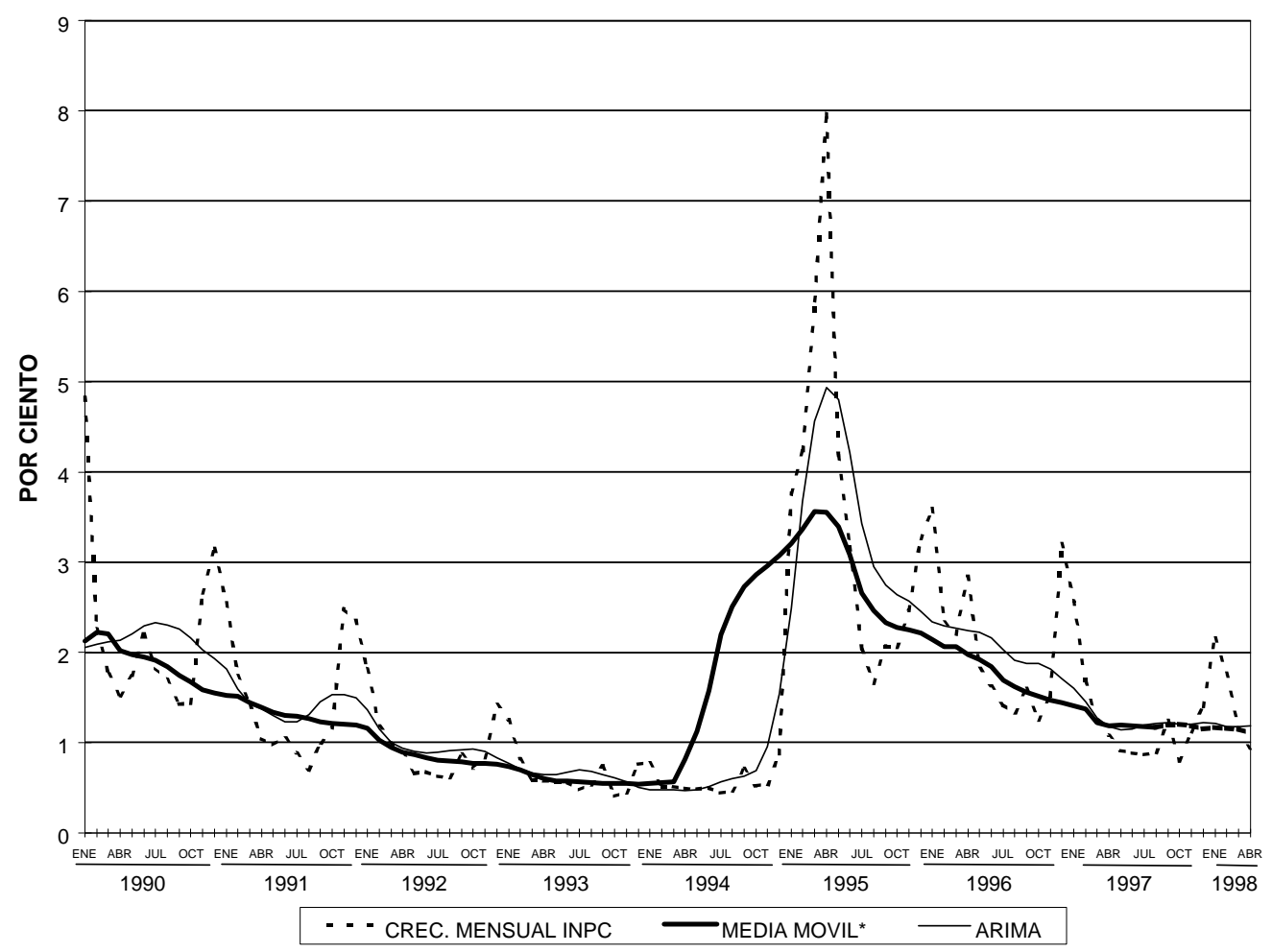

* A partir de septiembre de 1997 la media móvil centrada se calculó utilizando pronósticos del INPC.

Es importante señalar que, por construcción de la media móvil, los cambios bruscos y repentinos del crecimiento del INPC perduran más y se anticipan al periodo en el que suceden, como por ejemplo, los observados durante el primer semestre de 1995. En este sentido, en los periodos previos a los cambios extremos la media móvil sobrestima el movimiento tendencial y en los periodos posteriores, lo subestima. La tendencia extraída con un modelo ARIMA es más flexible para captar la dimensión temporal de los cambios en la inflación. No obstante, en periodos de relativa estabilidad ambas medidas convergen (por ejemplo, durante 1992 y 1993). Si, como lo muestra la experiencia de otros países, el proceso inflacionario en México siguiera un patrón sin cambios bruscos, las dos medidas mostrarían una tendencia similar. ${ }^{12}$

Dado el patrón de cambios de la inflación en México, puede concluirse que para este caso, un modelo ARIMA es más conveniente para extraer la tendencia del crecimiento mensual del INPC, por lo que en este documento se considerará como inflación subyacente a la tendencia obtenida con este procedimiento. No obstante, cabe destacar que a partir de enero de 1997 las dos medidas vuelven a converger, por lo que en ausencia de nuevos cambios abruptos en el crecimiento mensual del INPC, la media móvil centrada puede ser un procedimiento más práctico para calcular la inflación subyacente. En general, la experiencia internacional indica que de perdurar la citada similitud entre las tendencias que resultan de estas dos técnicas se opta por la media móvil centrada ya que es la más simple de calcular y la que es más fácil de explicar al público en general.

\footnotetext{
${ }^{12}$ Espasa et al (1987) encuentran que para la economía española las dos tendencia son muy similares.
} 
Con la inflación subyacente es posible determinar si el ritmo de crecimiento de los precios está acelerándose, desacelerándose o si se ha estabilizado en un cierto nivel. La inflación subyacente puede ser utilizada para valorar los procesos de aceleración o desaceleración de los precios al comparar la tendencia que se genera al incorporar nuevas observaciones con la que se obtuvo sin ellas. De manera que si se detectan desviaciones sistemáticas positivas se puede concluir que el proceso inflacionario está acelerándose.

En la gráfica 1 se observa que a partir de 1990 y hasta finales de 1994 la inflación tiene una tendencia decreciente. Posteriormente, a mediados de 1994 esta tendencia se revierte ligeramente y crece abruptamente durante el primer trimestre de 1995. Finalmente, a partir de abril de 1995 la inflación vuelve a mostrar una clara tendencia decreciente. Sin embargo, también se observa que a partir de abril de 1997 dicha tendencia decreciente se debilita.

\section{NÚCLEO IN FLACIONARIO}

El núcleo inflacionario se refiere a la tasa de crecimiento porcentual de un índice de precios que en su construcción elimina algunos de los bienes y servicios incluidos en el IPC cuyos precios son, por lo general, los más volátiles. Se presume que la inflación obtenida a partir de este índice mostrará un comportamiento más estable en el tiempo.

Como se mencionó en la sección II, el núcleo inflacionario fue una de las primeras medidas ideada por algunos bancos centrales para depurar al IPC de cambios en precios relativos. En la literatura económica no se registra ningún criterio teórico o estadístico para seleccionar los bienes y servicios que se excluyen. Al parecer esta selección ha dependido de la percepción particular sobre los precios más variables o del tipo de choques de precios relativos que cada país ha podido identificar. Así, por ejemplo, en los Estados Unidos de América se definió a la inflación núcleo como el índice de precios que excluye la energía y los alimentos; en Japón, el índice que excluye los precios de los alimentos frescos; en Canadá se excluye la energía, los alimentos y los impuestos indirectos; en Nueva Zelandia se utiliza al índice que excluye pagos de intereses; y en el Reino Unido se utiliza el índice de precios al menudeo que excluye pagos de intereses sobre préstamos hipotecarios.

México no ha sido la excepción en cuanto a la construcción de índices que omiten algunos bienes y servicios, pero ello, en general, no ha obedecido a una intención por conocer el crecimiento tendencial del nivel general de precios, sino que han sido elaborados para estudiar otros fenómenos económicos particulares. En México, a los índices que contemplan subgrupos específicos del INPC se les conoce como Indies Espeeiales a saber: el índice de la canasta básica; el índice de precios no incluidos en la canasta básica; el índice de precios comerciables; el índice de precios no comerciables; y, el índice general sin rentas y colegiaturas. ${ }^{13}$

El índicedela canasta básica tuvo relevancia a partir de 1987 con el fin de analizar el comportamiento de los precios de aquellos bienes y servicios que estaban controlados bajo el Pacto de Solidaridad. ${ }^{14}$ El complemento de este índice, el índicedebienes noinduidos en la canasta básica incluye los bienes y servicios cuyos precios se determinaban libremente en el mercado. Por otra parte, el índicedepreios comeriables se diseñó como un indicador para reflejar el comportamiento de las expectativas del tipo de cambio y sólo

\footnotetext{
${ }^{13}$ Estos índices son elaborados y publicados mensualmente por el Banco de México, en los "Indicadores Económicos” y en el "Cuaderno Mensual de Índices de Precios"

${ }^{14} \mathrm{El}$ pacto de solidaridad económica fue el arreglo institucional entre los diversos sectores económicos del país que constituyó uno de los componentes principales del plan de estabilización establecido a finales de 1987 y que con ciertas modificaciones perduró hasta 1997. Mediante estos pactos se determinaban lineamientos para la política económica y se comprometía a los participantes en cuanto a los niveles máximos de incrementos en los precios, salarios y el tipo de cambio.
} 
incluye el precio de bienes comerciables en el exterior, mientras que el índie de preios mo comerables incluye el resto de los bienes y servicios.

Finalmente, el índiegeneal sin rentas y degjaturas es el único índice especial que fue elaborado con el objetivo de eliminar distorsiones transitorias en los precios debidas a la estacionalidad o cambios bruscos de las rentas y las colegiaturas. Sin embargo, este índice no se ha utilizado como el indicador de inflación, muy probablemente, por lo específico de los precios que se eliminan.

La inflación núcleo tiene la ventaja de ser un concepto muy intuitivo, de fácil comprensión y aceptación para el público en general. Como se mencionó anteriormente, en la literatura no se ha propuesto un criterio para seleccionar cuántos y cuáles de los bienes y servicios que componen el IPC se deben excluir para estimar la inflación núcleo. En este trabajo se presenta una propuesta metodológica para realizar esta selección.

El objetivo es determinar con base a un criterio estadístico, aquellos bienes y servicios cuya inflación presenta una mayor volatilidad. Sin embargo, como las inflaciones de estos productos no son estacionarias, ciertos conceptos de volatilidad como la varianza no son válidos. Existen diferentes posibilidades para determinar un criterio de volatilidad de una serie no estacionaria, desde modelos ARCH y GARCH hasta procedimientos más simples con base en las desviaciones de la serie respecto a su tendencia 0 a otra serie con la que comparta el proceso tendencial. ${ }^{15}$

En el presente estudio se optó por medir la variabilidad de los crecimientos porcentuales mensuales de cada uno de los bienes y servicios que componen al IPC con base en su desviación respecto a una medida tendencial común. Para ello se consideró el estimador estadístico conocido como error cuadrático medio con respecto a la inflación subyacente. ${ }^{16}$ Una vez conocidos estos indicadores de variabilidad, las series se ordenan para determinar los bienes y servicios cuyos cambios de precios son más volátiles.

El siguiente paso es determinar un criterio para definir cuántas de las series más volátiles se deben eliminar para el cálculo del nuevo índice. Una opción sería eliminar los elementos de mayor variabilidad hasta que se agote cierta proporción del ingreso gastado en esos bienes y servicios. Sin embargo, esta proporción continuaría siendo arbitraria. ${ }^{17}$

En este documento se propone un criterio para determinar dicha proporción: ${ }^{18}$

1. Se eliminan las series de los bienes y servicios más volátiles hasta que la proporción del ingreso gastado en los bienes eliminados sea 1\%, $5 \%, 10 \%$, etc.

2. Se estiman índices de precios "núcleo" con los elementos no eliminados a cada proporción del gasto, en cada caso se ajustan los ponderadores para que su suma sea uno.

3. Se calcula el error cuadrático medio respecto a la inflación subyacente de cada una de las inflaciones reportadas por estos índices "núcleo". Se determina que el núdø inflacionario es la estimación que minimiza este indicador de dispersión. ${ }^{19}$

\footnotetext{
${ }^{15} \mathrm{ARCH}$ es un modelo de heterocedasticidad condicional autorregresiva y GARCH es el modelo de ARCH generalizada. Los modelos ARCH y GARCH son utilizados para modelar series que presentan cambios de su varianza en el tiempo. Para un tratamiento formal de estas metodologías ver, Engle (1982) y Bollerslev (1986).

${ }^{16}$ En principio es posible medir las desviaciones de cada serie respecto de su propia tendencia, sin embargo, este procedimiento no provee de información adicional significativa que justifique el esfuerzo de seleccionar un modelo para determinar la tendencia de cada bien. Adicionalmente el obtener los errores cuadráticos medios de cada serie con respecto a una tendencia común permite que éstos sean directamente comparables.

${ }^{17}$ La proporción del ingreso gastado en cada bien o servicio queda determinada por su ponderador en el IPC.

${ }^{18} 0$ tra opción sería eliminar un cierto número de las series más volátiles, pero de igual manera el citado número sería arbitrario. En todo caso es posible utilizar el criterio propuesto para determinar dicho número.
} 
Este procedimiento para seleccionar los elementos que históricamente han impregnado de una mayor volatilidad al índice de precios, al incorporar un criterio estadístico, elimina la arbitrariedad en la determinación de los bienes y servicios a excluir en el cálculo de núcleo inflacionario. Esta metodología permite estimar una medida de inflación que elimina los cambios en precios relativos más comunes y que sirve como un indicador no paramétrico y de cálculo sencillo de la tendencia inflacionaria. Desafortunadamente, como el error cuadrático medio es un indicador que depende del periodo muestral considerado, es importante continuar evaluando los elementos más volátiles cada cierto intervalo de tiempo.

En particular, para calcular este índice para México la tendencia considerada fue la inflación subyacente del modelo ARIMA. ${ }^{20}$ De acuerdo al procedimiento descrito se calculó la volatilidad del cambio porcentual con respecto al mes inmediato anterior de los precios de cada uno de los 313 bienes y servicios que conforman el INPC ${ }^{21}$, y se ordenaron de mayor a menor. Se fueron eliminando los bienes y servicios más volátiles hasta que se agotaba un punto porcentual del ingreso gastado en ellos, a lo cual se recalculaba un nuevo índice con los elementos que no se eliminaban. Al comparar el error cuadrático medio del crecimiento porcentual mensual de cada uno de los índices obtenidos, se determinó que este indicador se minimiza para el índice que elimina bienes y servicios cuya participación en el gasto asciende a 44 por ciento. ${ }^{22}$ De manera que, en adelante, el crecimiento porcentual mensual correspondiente a dicho índice se considerará como el núdeøinflacionario

En la gráfica 2 se presenta la relación entre el error cuadrático medio y el porcentaje del ingreso que representan los bienes eliminados. Como ahí se observa, al eliminar los bienes que equivalen a una pequeña porción del ingreso se logra disminuir el error cuadrático medio, pero esto es sólo un mínimo local que se revierte rápidamente. El mínimo global de dicha relación se encuentra hasta el nivel de 44 por ciento mencionado previamente.

\footnotetext{
${ }^{19}$ Este criterio para determinar el porcentaje óptimo es similar al utilizado para determinar el nivel de truncamiento óptimo de los estimadores de influencia limitada. Véase Bryan, Cecchetti y Wiggins (1997) para mayor referencia.

${ }^{20}$ También se realizaron pruebas utilizando la tendencia obtenida con la media móvil. Los resultados obtenidos con estas pruebas no fueron cualitativamente diferentes.

${ }^{21} \mathrm{~A}$ este respecto conviene aclarar que el INPC se compone de 313 bienes y servicios a partir de la nueva metodología de 1994. Anteriormente, el INPC se conformaba por un número menor de bienes y servicios. En este trabajo las series no existentes en la metodología previa a 1994 se generaron asignándoles el índice de precios del producto genérico más cercano en su definición. Se optó por esta reconstrucción de las series para utilizar los ponderadores más recientes para toda la muestra.

${ }^{22}$ Los errores cuadrático medios con los cuales se determinaron los bienes que se eliminaron se obtuvieron sólo para el periodo que abarca la nueva metodología del INPC.
} 


\section{Gráfica 2 \\ RELACIÓN ENTRE EL ERROR CUADRÁTICO MEDIO Y EL PORCENTAJE DEL INGRESO QUE REPRESENTAN LOS ELEMENTOS ELIMINADOS}

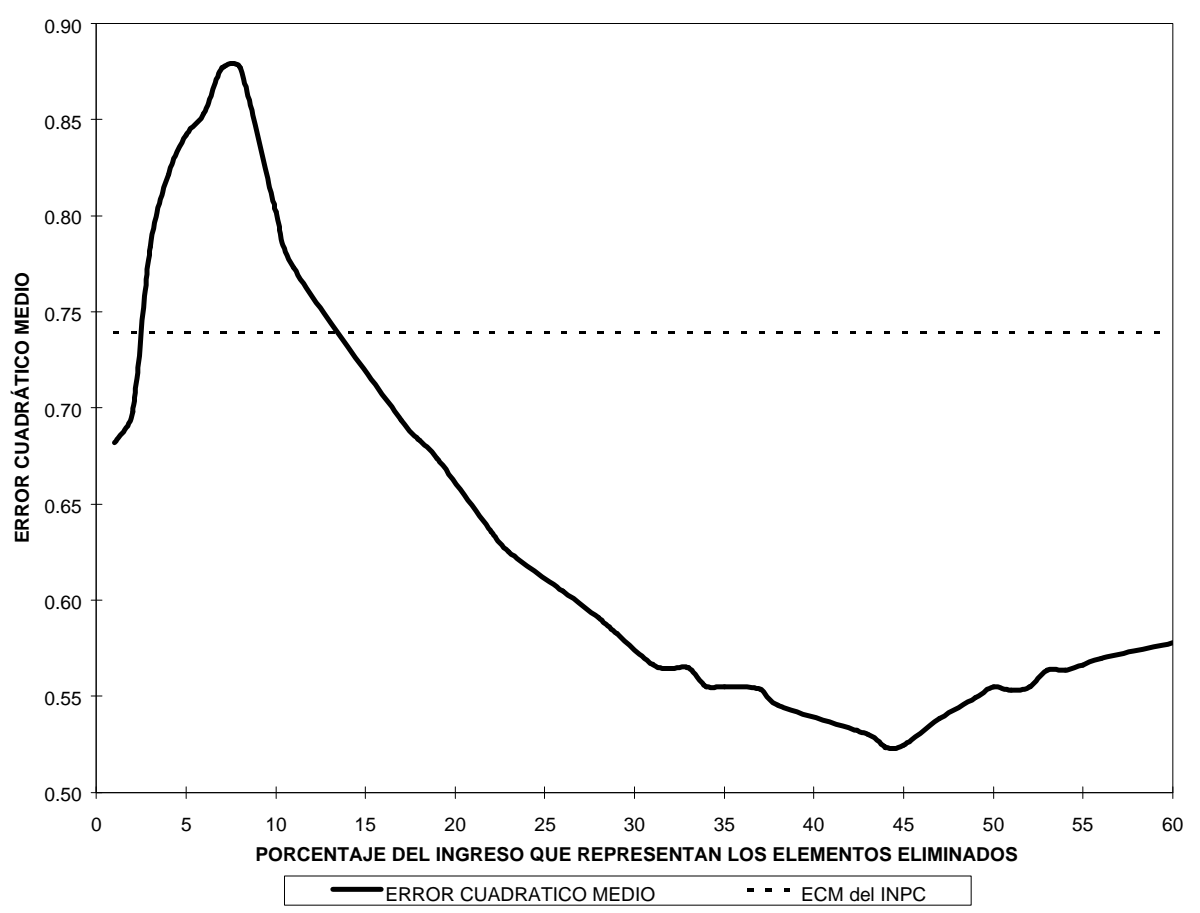

Cabe destacar que dicho porcentaje corresponde a eliminar los precios de 123 bienes y servicios, principalmente, a los alimentos no procesados (chile poblano, tomate verde, limón, entre otros), la energía (gasolina, electricidad y gas doméstico), las colegiaturas, el transporte (taxi, autobús, metro, y gastos de automóvil particular) y el servicio telefónico (local y larga distancia). ${ }^{23}$ Los bienes y servicios eliminados son aquéllos que sus precios, en general, se ven influidos por factores estacionales (bienes agrícolas), han estado sujetos a controles administrativos (leche, huevo, tortilla, metro, colectivo, gasolina, gas, teléfono, entre otros) o que se ajustan sólo esporádicamente (colegiaturas, refrescos).

Llama la atención que este procedimiento resulta en la eliminación de algunos de los bienes que tradicionalmente se han excluido en otros países. Asimismo cabe destacar que no se elimina ningún componente de rentas o de vivienda de manera que, bajo este criterio, el ímoice general sin rentas ni clejaturas no sería un buen indicador del núcleo inflacionario. Por último, es importante mencionar que de haberse utilizado el criterio de eliminación basado en un porcentaje arbitrario del gasto se podría haber obtenido una medida del proceso tendencial menos acertada que el mismo crecimiento del INPC.

Es posible obtener información sobre algunos aspectos del proceso inflacionario de corto plazo al comparar el núcleo inflacionario con la variación porcentual del INPC. Para ello, es necesario considerar tanto la dirección como la magnitud de los cambios en ambas medidas de inflación. Si el cambio en el núcleo inflacionario es menor (mayor) que el del INPC, se presume que el INPC está reflejando un cambio en precios relativos. Es decir, que el crecimiento de los precios de algunos de los elementos que fueron excluidos del INPC para la construcción del núcleo inflacionario aumentó (disminuyó) en relación con el crecimiento del nivel general de precios. En la gráfica 3 se compara al núcleo inflacionario con el cambio porcentual del INPC.

\footnotetext{
${ }^{23}$ En el Apéndice 1 se presenta una lista completa de los bienes y servicios excluidos.
} 
Antes de 1995, la variación mensual del INPC oscila con mayor frecuencia alrededor del núcleo inflacionario, lo que se atribuye a cambios frecuentes en los precios relativos de los bienes cuyos precios han sido los más volátiles. Por otra parte, a partir de 1995 las dos medidas de inflación siguen un patrón de movimientos similar con poca divergencia, lo que indica una menor variabilidad en precios relativos (esto puede atribuirse en buena medida a la modificación en la estrategia de determinación de precios administrados). ${ }^{24}$

Se puede comprobar que el mayor crecimiento del INPC que se observa típicamente en el mes de septiembre a partir de 1992, corresponde a un cambio estacional en precios relativos (en particular, al aumento de las colegiaturas), ya que la inflación medida a través del núcleo inflacionario no presenta este incremento típico.

\section{Gráfica 3 \\ NUCLEO INFLACIONARIO}

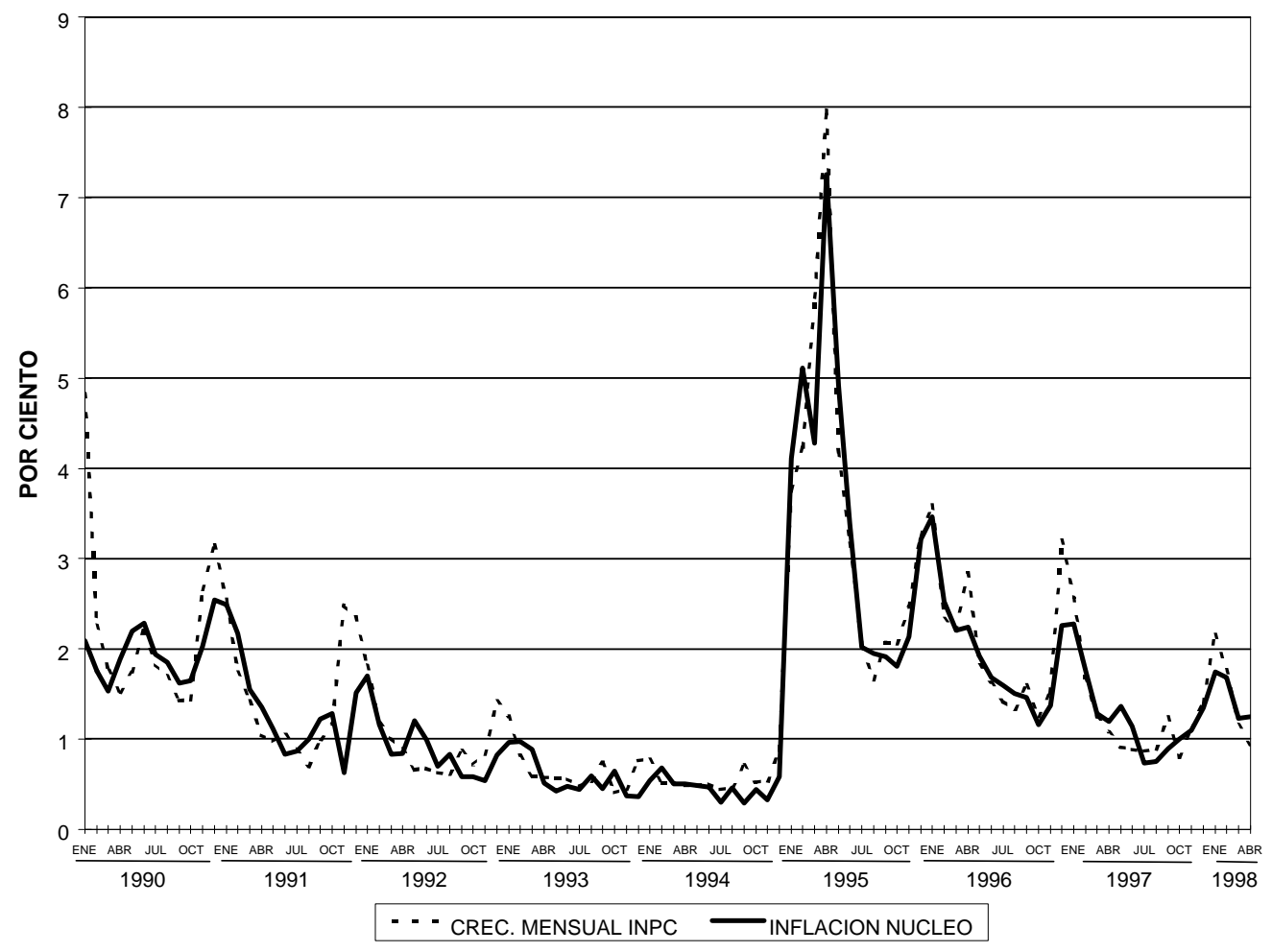

De lo anterior se concluye que el núcleo inflacionario logra capturar el cambio en el nivel de precios que no es atribuible a las principales variaciones en precios relativos debidas a factores estacionales 0 a asincronía en la fijación de algunos precios. Por otro lado, la crítica principal que puede hacerse a esta medida de inflación es que no existe una razón por la cual excluir del IPC para todo el periodo muestral a los mismos bienes y servicios, ya que se corre el riesgo de que en ciertos periodos estos bienes eliminados contengan información importante sobre el fenómeno inflacionario. También es posible que durante ciertos lapsos del periodo muestral algunos bienes y servicios no excluidos en la inflación núcleo hayan presentado (o presenten en el futuro) variaciones atípicas respecto a la tendencia o cambios en precios relativos importantes. Para estos periodos de la muestra la inflación

\footnotetext{
${ }^{24} \mathrm{G}$ amboa (1997) reseña las modificaciones a la política de precios administrados en el periodo de 1985 a 1997.
} 
núcleo no sería representativa de los cambios en el nivel general de precios. En este sentido, se puede concluir que el núcleo inflacionario es una medida poco flexible para incorporar cambios en la formación de precios de la economía.

\section{MEDIDAS DE INFLACIÓN CON ESTIMADORES DE INFLUENCIA LIMITADA}

Las medidas de inflación con estimadores de influencia limitada también buscan obtener una medida de inflación que no refleje modificaciones en precios relativos o choques exógenos mediante la elaboración de un nuevo índice que excluye algunos de los precios de los bienes y servicios contemplados en el IPC. Sin embargo, en contraste con el núcleo inflacionario que en cada mes elimina los mismos bienes y servicios del IPC, en este caso se propone un criterio mediante el cual en cada mes se eliminan los precios de distintos bienes y servicios en función del cambio en sus precios relativos.

A este respecto, resulta útil concebir los cambios en los precios de cada uno de los bienes y servicios que forman al IPC en un momento dado como la suma de: (i) el cambio en el nivel general de precios, y (ii) una perturbación específica al bien o servicio en cuestión, que origina un cambio en su precio relativo respecto al nivel general de precios. D e manera que:

$$
\pi_{\mathrm{i}}=\pi+\varepsilon_{\mathrm{i}}
$$

donde $\pi_{\mathrm{i}}$ es la inflación de cada bien o servicio " $\mathrm{i}$ ", $\pi$ es el cambio en el nivel general de los precios y $\varepsilon_{\mathrm{i}}$ es una perturbación específica sobre el precio del bien o servicio "i". D e esta forma, los cambios en los precios de todos los bienes y servicios que componen al IPC en un mes en particular se distribuirán conforme a las perturbaciones específicas a cada bien.

Por extensión, es de esperarse que los bienes y servicios cuyos precios no estuvieron sujetos a perturbaciones específicas sólo aumenten conforme al nivel general y que sus cambios se concentren en la parte central de la distribución. En consecuencia, si se dispone de una medida que represente la parte central de esta distribución, se conocerá el crecimiento de los precios que hubiera prevalecido en ausencia de cambios en precios relativos.

Para este propósito se ha sugerido la utilización de la media truncada 0 de la mediana. ${ }^{25}$ La media truncada equivale a la media que se obtiene cuando se eliminan del cálculo una proporción de los valores más altos y los más bajos de la distribución estadística de los cambios en precios. Este indicador aminora el peso de los valores extremos de una distribución estadística (se limita la influencia de las colas de la distribución), la mediana corresponde a la media truncada en la que se elimina el 50\% de la distribución en cada cola.

Es importante señalar que la distribución de los cambios en los precios de los bienes y servicios que componen el IPC en cada periodo se construye con base a la fracción del ingreso destinada al gasto en ese bien. En otras palabras se supone que en dicha distribución de probabilidad, la probabilidad del cambio en el precio de cada elemento del índice es igual a su ponderador. De esta forma, eliminar un cierto porcentaje de las colas de la distribución, equivale a excluir una determinada proporción del gasto en los bienes cuyos crecimientos porcentuales se ubican en los extremos inferior y superior. Por ejemplo, la media truncada al 10\% implica excluir del índice los bienes y servicios con mayor y menor cambio porcentual que representan el $5 \%$ del gasto en cada caso. Una vez eliminados los elementos cuyos cambios en precios son extremos, se reponderan las fracciones del ingreso destinado al gasto de los elementos restantes.

\footnotetext{
${ }^{25}$ Bryan y Cecchetti (1993).
} 
En particular, para el caso de México, se calculó para cada mes de la muestra el cambio porcentual con respecto al mes inmediato anterior de los precios de cada uno de los 313 bienes y servicios que conforman el INPC. ${ }^{26}$ D e la distribución estadística de estos cambios, se eliminaron simétricamente de cada cola los cambios en los precios de los bienes y servicios que correspondían a 2.5, 5, 7.5, etc., y hasta 50 por ciento por cola del ingreso gastado en ellos. Posteriormente, se recalculó un nuevo índice con los precios de los bienes y servicios no eliminados a cada porcentaje de truncamiento.

El nivel de truncamiento óptimo se obtuvo siguiendo un criterio similar al empleado con la inflación núcleo. Para cada serie de crecimientos porcentuales mensuales a cada nivel de truncamiento ${ }^{27}$ se obtuvo el error cuadrático medio con respecto a la tendencia del crecimiento mensual del INPC y se seleccionó el nivel de truncamiento que minimizaba dicho error. ${ }^{28}$

En la gráfica 4 se dibuja la relación entre el error cuadrático medio y los distintos niveles de truncamiento. Como ahí se observa, la relación es altamente no lineal pero siempre da un error cuadrático medio inferior al del INPC (que corresponde a valor cuando el nivel de truncamiento es cero), y posee un mínimo global en el nivel de truncamiento 5.5 por ciento del área de la distribución por cola. Esta forma es congruente con la reportada para los Estados Unidos de América en Bryan, Cecchetti y Wiggins (1997). Es importante destacar que la mediana -que en la gráfica corresponde a truncar el 50 por ciento de la distribución- no resulta en el menor error cuadrático medio.

\footnotetext{
${ }^{26}$ Ver nota 21.

${ }^{27} \mathrm{No}$ es posible calcular directamente el crecimiento porcentual mensual del nuevo índice de precios ya que en cada periodo se eliminan distintos bienes y servicios. Por lo que es necesario construir una serie de los crecimientos mensuales de índices comparables. Para ello, en cada periodo, a la vez que se calcula el nuevo índice para el mes corriente, se calcula un índice que incluye a los mismos elementos para el mes anterior y se obtiene, entonces, el crecimiento porcentual relevante para dicho mes.

${ }^{28}$ Ver Bryan, Cecchetti y Wiggins (1997).
} 


\section{Gráfica 4 \\ RELACIÓN ENTRE EL ERROR CUADRÁTICO MEDIO Y EL PORCENTAJE DE LA DISTRIBUCIÓN TRUNCADA EN CADA COLA}

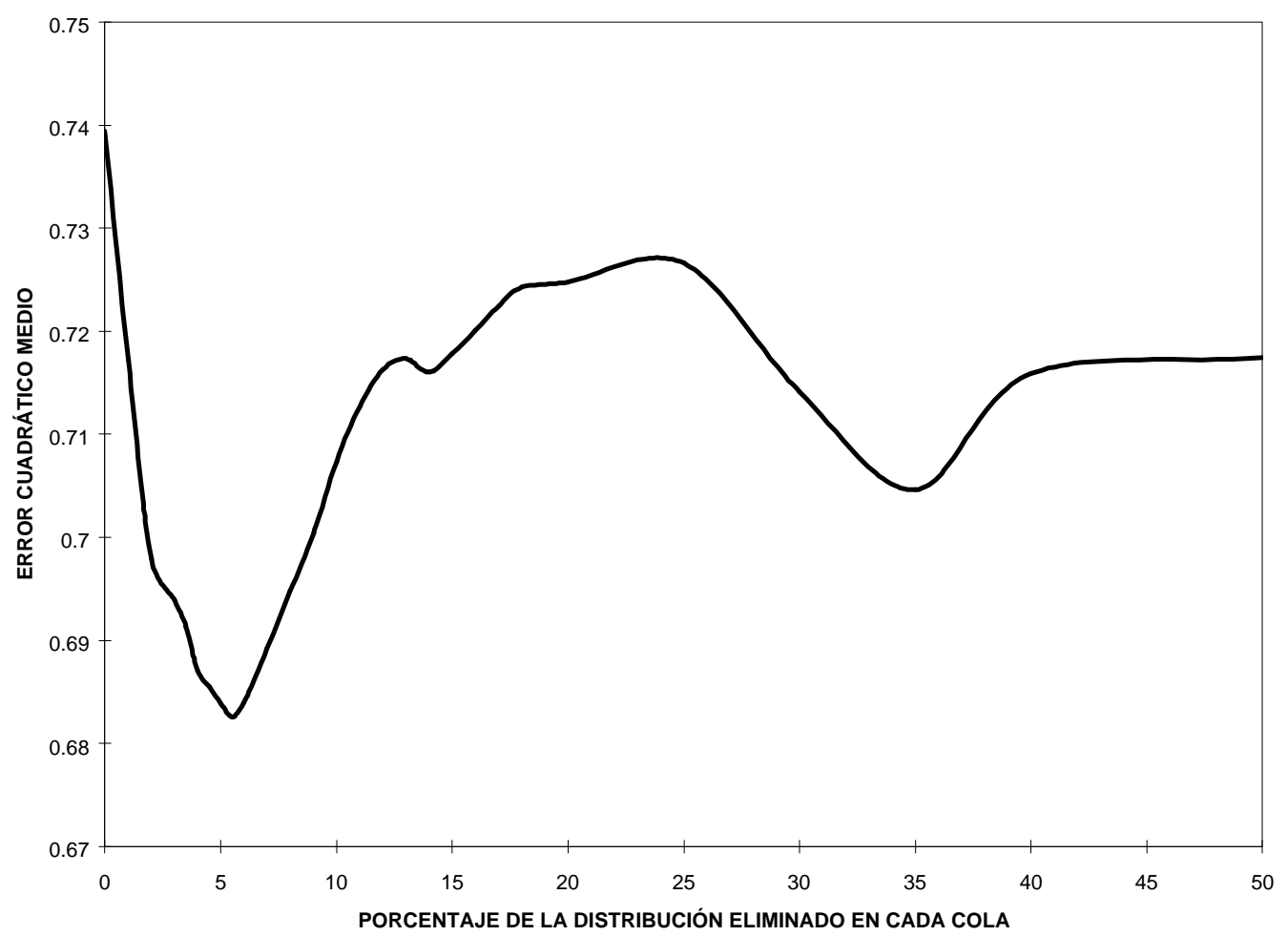

Como el objetivo de la medida es eliminar los mayores cambios en precios relativos, su interpretación es similar a la realizada con el núcleo inflacionario, aunque en este caso hay mayor certidumbre de que se eliminan los principales cambios en precios relativos en cada periodo.

En la gráfica 5 se compara al cambio mensual del INPC con la inflación medida por la media truncada al 11 por ciento y por la mediana, respectivamente. ${ }^{29}$

Con respecto a la inflación calculada con la media truncada al 11\%, en el panel superior de la gráfica 5 se pueden distinguir, al igual que con la inflación núcleo, dos patrones de comportamiento diferentes, uno para el periodo previo a 1995 y otro para periodo posterior a esa fecha. Antes de 1995 el patrón de movimientos de esta medida difiere en múltiples ocasiones del patrón de movimientos del crecimiento mensual del INPC, lo cual puede ser indicativo de variaciones frecuentes de precios relativos. En contraste, a partir de 1995, el patrón de movimientos de ambas medidas coincide en la mayoría de los casos, lo que evidenciaría una menor variabilidad de precios relativos. Este cambio en la variabilidad de precios relativos podría deberse a modificaciones en la política de fijación de precios administrados.

Al igual que con la inflación núcleo, en el citado panel de la gráfica 5 se observa que, el aumento estacional del crecimiento del INPC durante el mes de septiembre se debe a cambios de precios relativos ya que en la inflación medida con la media truncada dicho aumento prácticamente no se

\footnotetext{
${ }^{29}$ En el apéndice 2 se presenta una relación de la frecuencia con la que cada componente del INPC es excluido de la media truncada al $11 \%$.
} 
presenta. En particular, en cada septiembre a partir de 1992 la inflación de la media truncada siempre es menor que el crecimiento del INPC. El referido cambio de precios relativos muy probablemente sea causado por el efecto sobre las colegiaturas del inicio del ciclo escolar.

Un aspecto a destacar de la comparación entre estas dos medidas es que en los periodos con incrementos bruscos en la inflación, el crecimiento del INPC se sitúa, por lo general, por encima de la inflación calculada con la media truncada. Este resultado podría indicar que en periodos de aumento repentino en los precios, se presentan normalmente cambios en precios relativos que sesgan el incremento del INPC.

Llama la atención que la inflación medida con la media truncada se ubica, en general, durante la mayoría del periodo considerado, pero particularmente después de 1995, por debajo del crecimiento mensual del INPC. Muy probablemente esto sea resultado de que la distribución de los cambios de los precios de los bienes y servicios que componen al INPC es asimétrica y se encuentra sesgada hacia arriba.

En el panel inferior de la gráfica 5 se observa que el comportamiento de la inflación medida con la mediana es muy similar al descrito para la media truncada al $11 \%$ de manera que se mantienen, en términos generales, las mismas conclusiones. Sin embargo, es importante destacar que con esta medida es más marcado que sus niveles se encuentran en general, y particularmente después de 1995, por debajo del crecimiento mensual del INPC

\section{Gráfica 5}

\section{MEDIDAS DE INFLACIÓN CON ESTIMADORES DE INFLUENCIA LIMITADA}

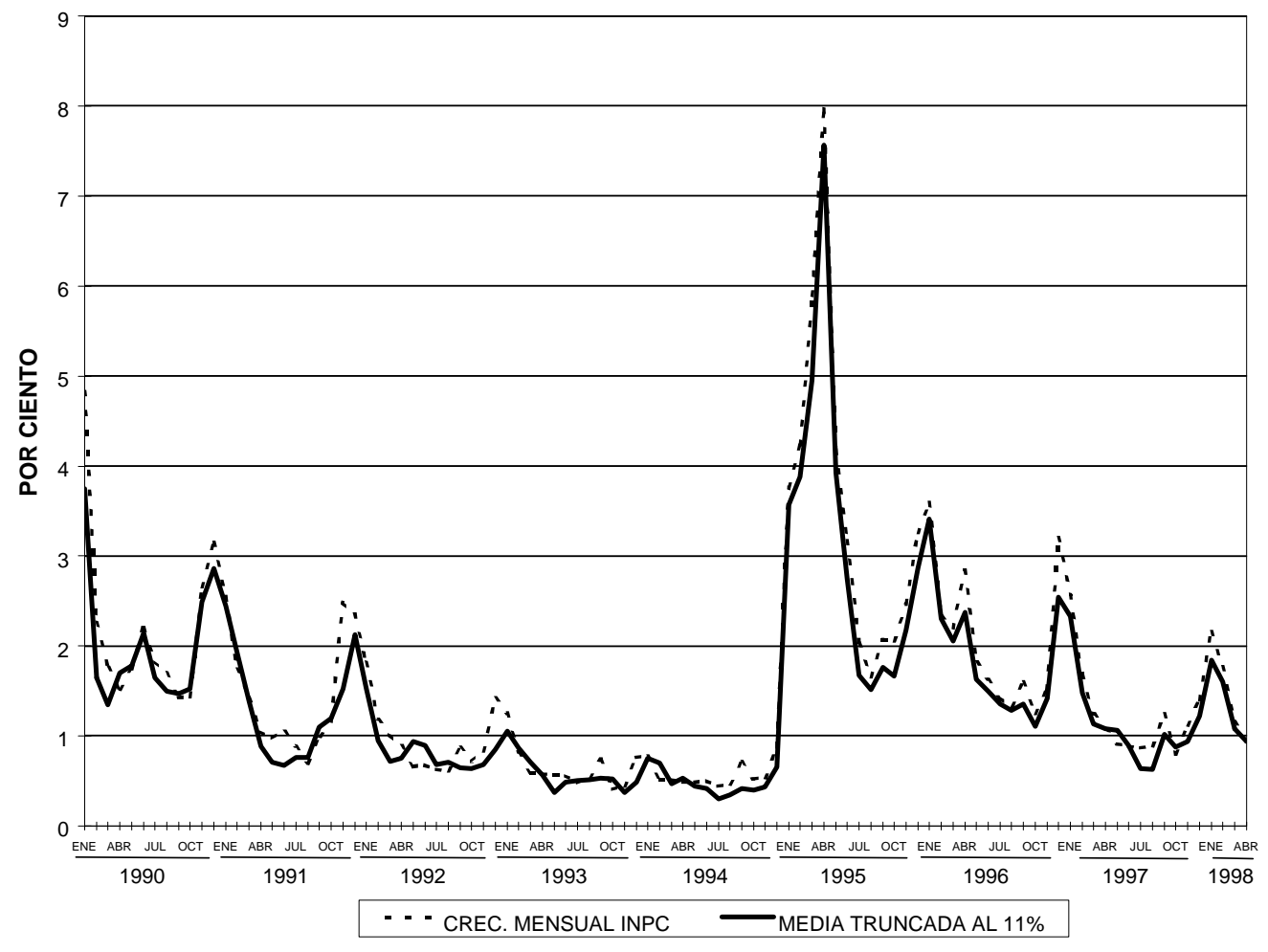




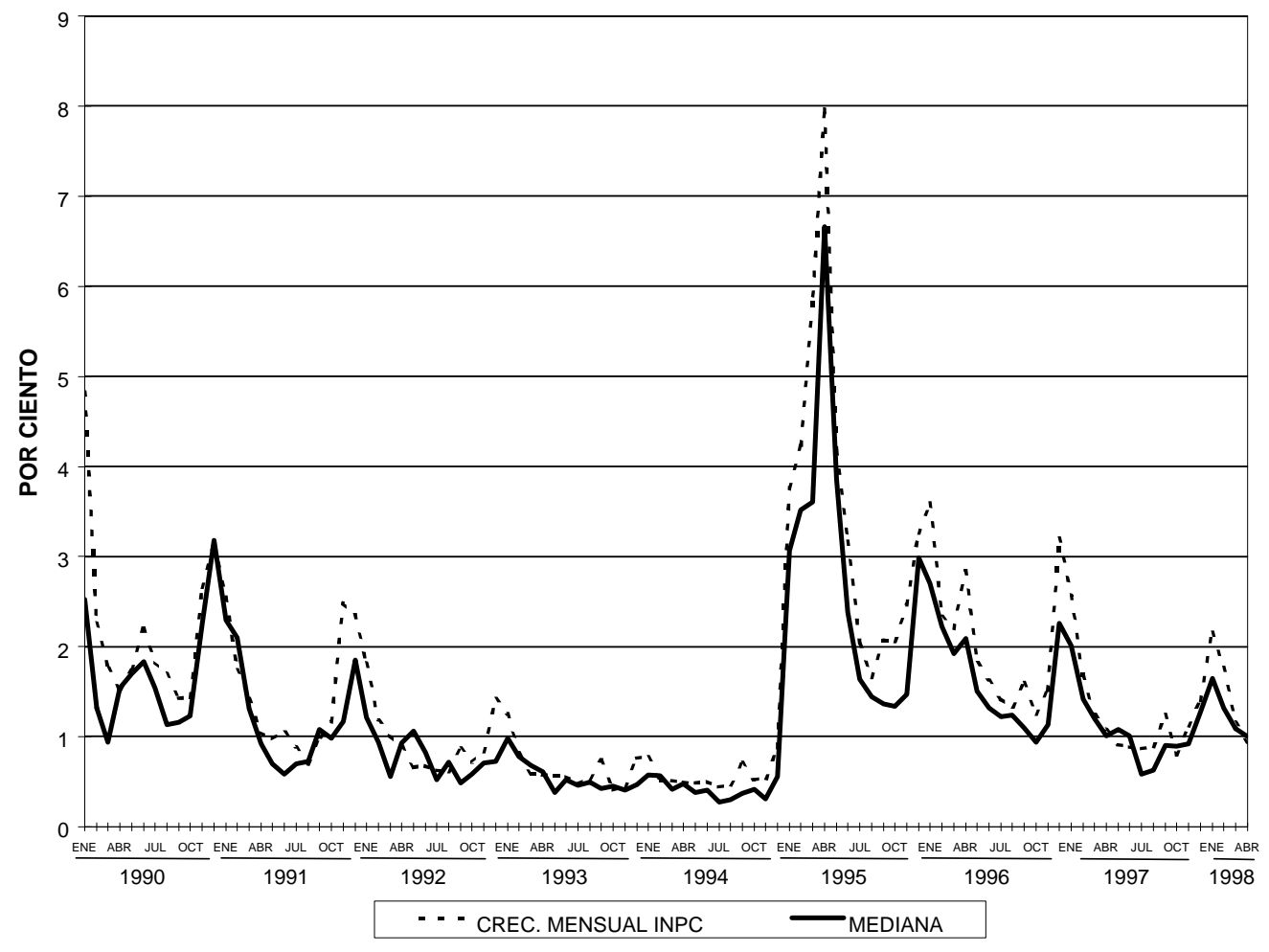

\section{INFLACIÓN LATENTE}

Las medidas de inflación expuestas en las secciones anteriores tienen como finalidad común obtener de la información del IPC un indicador de inflación libre de los cambios en los precios relativos, ya sea mediante la extracción de la tendencia del crecimiento de este índice 0 eliminando algunos de sus componentes. Sin embargo, también sería deseable contar con una medida de inflación que diera mayor información sobre el origen de las perturbaciones que generan cambios en los precios. En particular, una medida de aquella parte del cambio en el nivel general de precios susceptible de ser influida por la política monetaria, aportaría información valiosa para analistas y autoridades.

La inflación es en el largo plazo un fenómeno puramente monetario y como tal no debería tener efecto sobre el nivel de actividad económica de largo plazo. Si bien en el corto plazo pueden existir ciertas rigideces en precios y salarios que tengan un efecto sobre la producción, es generalmente aceptado que una vez que transcurre el tiempo necesario para que los contratos sean renegociados, los movimientos en los precios significan únicamente incrementos en magnitudes nominales sin un efecto sobre la economía real.

El nivel general de precios puede verse afectado por presiones de demanda o presiones transitorias de oferta, durante el ciclo económico, y también por perturbaciones que afectan al nivel de producción de largo plazo. En este último caso por ejemplo, un choque de oferta de largo plazo generará un incremento en los precios independientemente de la postura de la política monetaria y de las otras presiones de demanda. Sería útil contar con una medida de inflación que identificara el aumento en el nivel general de precios que no tiene un impacto en el mediano y lango plazo sobre la economía real, generando únicamente movimientos nominales en el largo plazo. Este indicador permitiría analizar las presiones inflacionarias susceptibles de ser afectadas por las políticas de demanda agregada. 
Quah y Vahey (1993) señalan que el problema de utilizar el crecimiento porcentual del IPC como medida de inflación va más allá de un error de medición y se relaciona con una deficiencia de la medida tradicional de inflación para representar a su contraparte teórica. ${ }^{30}$ Estos autores proponen una metodología para estimar una medida de inflación que incorpora en su construcción una de las propiedades de largo plazo del concepto teórico de inflación, la clásica dicotomía entre variables nominales y reales.

Su metodología (ver apéndice 3) supone que existen dos tipos de perturbaciones que afectan al crecimiento del nivel general de precios y al producto, el primer tipo de perturbaciones no tiene un efecto de largo plazo sobre la producción, mientras que el segundo tipo recoge a las perturbaciones que afectan el nivel potencial o de largo plazo de la actividad económica. ${ }^{31}$ D e esta forma, descomponen la inflación ${ }^{32}$ en su componente latente, que es aquel asociado con las perturbaciones que no tienen un efecto de largo plazo sobre la producción y que se denominará inflación latente y en su componente no-latente, resultado del segundo tipo de perturbaciones.

Los choques con un efecto de largo plazo sobre la producción generan cambios en la tasa natural de desempleo. Como la inflación latente elimina la parte del crecimiento de los precios asociada con estos choques, en el largo plazo no existirá sustitución entre esta inflación y el desempleo. En este sentido, la inflación latente es compatible con una curva de Phillips vertical en el largo plazo. Por otro lado, las perturbaciones cuyo efecto sobre el producto tiende a desaparecer son las que generan las desviaciones cíclicas del producto alrededor de su nivel potencial, por lo que la inflación latente se asocia, en el corto plazo, con la posición del ciclo económico.

Conviene subrayar que al definir así las perturbaciones, no es posible distinguir si se generan en el lado de la oferta, de la demanda o de choques puramente nominales. El nivel de producción de largo plazo puede verse afectado tanto por choques de oferta (por ejemplo, una caída sostenida en el precio internacional del crudo) como de demanda (por ejemplo, una reducción en la inversión pública). Asimismo, los cambios transitorios del nivel de producción pueden tener su origen en la oferta (por ejemplo, cambios transitorios en la productividad) o en la demanda (por ejemplo, modificaciones en la postura de la política monetaria). Sin embargo, la mayoría de los choques de oferta tienen un efecto permanente sobre el producto y la mayoría de los choques de demanda sólo generan una desviación de corto plazo de la producción respecto a su nivel potencial.

Cuando existen perturbaciones negativas (positivas) que afectan al nivel de producción de largo plazo, la inflaciónserá superior (inferior) a la inflación latente. Conforme se disipe el efecto sobre la inflación de estas perturbaciones, ésta tenderá a converger hacia la inflación latente.

Si bien la inflación latente puede cambiar por muchos factores distintos a la política monetaria, cambios en la política monetaria tendrán efectos sobre este componente latente. Un incremento en la inflación latente no necesariamente implica que la postura de la política monetaria se relaje, sino que existen choques de demanda agregada, de oferta agregada transitorios o choques nominales, como sería una devaluación que no afecta el tipo de cambio real de largo plazo, que incrementan los precios. Sin embargo, en este caso la política monetaria podría jugar un papel en contrarrestar el efecto sobre la inflacón generada por dichos choques. La postura de la política monetaria debe evaluarse, entonces, con respecto al contexto de las presiones inflacionarias que enfrenta la economía, la inflación latente,

\footnotetext{
${ }^{30}$ El núcleo inflacionario y las medidas de inflación con estimadores de influencia limitada presentadas en las secciones anteriores, pretenden corregir únicamente el "error de medida" contenido en el crecimiento porcentual del IPC, por lo que también estarían sujetas a la misma crítica.

${ }^{31}$ Para identificar estos componentes se requiere, además de la información de precios, información sobre el nivel de actividad económica.

${ }^{32}$ En adelante, inflacón sin calificativos se referirá al crecimiento del indicador del nivel general de precios.
} 
en principio, puede mostrar las presiones sobre las que las políticas del banco central pueden tener cierta influencia.

Es importante mencionar que la inflación latente, en contraste con las medidas expuestas en las secciones anteriores, no elimina los cambios en los precios relativos. Si el indicador de inflación ${ }^{33}$ utilizado para estimar la inflación latente esta contaminado por cambios en precios relativos, la inflación latente los incluirá en su mayoría. Esto es así porque los cambios transitorios en precios relativos no tiene un efecto de largo plazo sobre el producto. Por otra parte, la inflación latente no tiene como objetivo ser una medida de la tasa de inflación de largo plazo. Muchas de las perturbaciones que son neutrales respecto al producto de largo plazo generan cambios en la tasa de inflación que son sólo transitorios, por ejemplo, un cambio temporal en la tasa de crecimiento monetario no incide sobre la producción de largo plazo, pero tampoco genera un cambio permanente en la inflación. Es posible, como se describe en la siguiente sección, utilizar la metodología VAR estructural para determinar una inflación de largo plazo o "permanente".

La inflación latente y su comparación con el indicador de la inflacoón del que provenga, puede dar información muy relevante sobre el tipo de presiones inflacionarias a que está sujeta la economía. Si los cambios en la inflacoón son muy semejantes a los cambios en la inflación latente, ello indicaría una ausencia de choques de largo plazo sobre el producto y por lo tanto que el cambio en la inflación se debe a factores sobre los cuales puede influir la política monetaria.

Cuando la inflación se sitúa por encima de la inflación latente, ${ }^{34}$ se están presentando perturbaciones negativas sobre el producto de largo plazo (por ejemplo, un choque negativo de oferta). Bajo estas condiciones, pueden presentarse tres casos. $i$ ) La inflación latente se mantiene constante, en este caso la política monetaria, en el contexto de las condiciones de demanda y oferta de corto plazo, no es inflacionaria. Al disiparse el efecto de los choques de largo plazo sobre la producción, la inflacón regresará hacia la inflación latente. ii) Si la inflación latente está disminuyendo, las presiones de demanda y de oferta de corto plazo son compatibles con el abatimiento de la inflacón No obstante, en estas condiciones una política monetaria restrictiva puede imponer mayores costos sobre el nivel de actividad de corto plazo, haciendo más lenta la recuperación económica. iii) Por último, si la inflación latente está creciendo, también se están presentando presiones de demanda o de oferta de corto plazo que generan una presión inflacionaria adicional, la cual podría ser contrarrestada usando políticas que afecten el comportamiento de la demanda agregada.

Cuando se presentan choques positivos sobre el producto de largo plazo, se obtiene que la inflacón se incrementa menos que la inflación latente. Analizando los casos posibles del comportamiento de la inflación latente se tiene: i) Si la inflación latente permanece constante, la reducción en la inflacoón es temporal, cuando se disipen los efectos positivos la inflacónregresará al nivel de la inflación latente. En este caso, el banco central está perdiendo una oportunidad de disminuir la inflacón con un bajo costo sobre la producción. ii) Cuando la inflación latente también disminuye, es posible que el banco central esté aprovechando esta oportunidad o que existan otras condiciones que permitan este resultado. iii) Finalmente, cuando la inflación latente está aumentando y la inflacón disminuyendo, se presenta una situación en la que las presiones inflacionarias de demanda o transitorias de oferta están siendo revertidas por los choques positivos sobre el producto de largo plazo. Si las presiones son de demanda, se podría hablar de una situación de euforia con un incremento tanto del producto potencial

\footnotetext{
${ }^{33}$ En este trabajo se utiliza el crecimiento porcentual del IPC como un indicador dela inflación Sin embargo, en principio es posible extraer el componente latente de cualquier indicador de inflación, como los expuestos en las secciones anteriores.

${ }^{34} \mathrm{El}$ análisis que se presenta en esta parte del documento supone que es posible obtener el nivel de la inflación latente. Sin embargo, como se menciona más adelante, el procedimiento estadístico propuesto por Quah y Vahey para la inflación latente sólo permite estimar el cambio en la inflación latente y no su nivel. Para determinar su nivel es necesario hacer un supuesto adicional sobre la condición inicial de la inflación latente.
} 
como de la brecha del producto. Sin embargo, al disiparse este efecto positivo es posible que la inflación crezca al nivel de la latente. En este caso, si las autoridades monetarias sólo observaran la inflacón (el crecimiento del IPC) podrían tomar medidas de política económica erróneas, como sería relajar la postura de la política monetaria.

El análisis anterior muestra el tipo de información sobre el proceso inflacionario que es posible extraer de la inflación latente. En particular, la inflación latente permite conocer más sobre el origen de las presiones inflacionarias y sobre algunos costos u oportunidades que puede tener la política antiinflacionaria. Sin embargo, la inflación latente que se ha descrito hasta este momento corresponde a un prototipo ideal, por lo que los resultados que se obtienen con el estimador estadístico de esta inflación deben ser tomados con precaución.

El principal problema de estimación de la inflación latente es que los dos tipos de perturbaciones que afectan a la inflación y al producto no son directamente observables. Para identificar estas perturbaciones se requiere realizar una descomposición de las series observables. Q uah y Vahey (1993) utilizan la metodología de vectores autorregresivos estructurales (VARE) de Blanchard y Q uah (1989) para identificar las perturbaciones estructurales y descomponer la serie de inflación en sus componentes latente y no-latente. Las principales características de esta metodología son: ${ }^{35}$

Se utiliza un método multivariado que incorpora, además de la información sobre la tasa de crecimiento de los precios, información sobre la actividad real. Para identificar las perturbaciones estructurales se imponen restricciones estadísticas que suponen que los dos tipos de perturbaciones son independientes (ortogonales). Adicionalmente se impone una restricción proveniente de la teoría económica que aísla aquellas perturbaciones cuyo efecto sobre el producto tiende a desaparecer, de manera que las perturbaciones restantes son las que tienen efectos permanentes sobre la producción. Como la definición misma de la inflación latente se impone como una de las condiciones de identificación, por construcción la inflación latente estimada será la inflación que se observaría si solamente existieran choques cuyo efecto sobre el producto desaparece.

De acuerdo a la metodología descrita, la inflación latente se estimó para México utilizando al crecimiento porcentual mensual de INPC como indicador de la inflacoóny al Indice del Volumen de la Producción Industrial (IVPI) como indicador del producto para el periodo de 1986 a abril de $1998{ }^{36}$ Mediante las pruebas Dickey-Fuller y Phillips-Perron se determinó que ambas series son I(1), de manera que ambas fueron diferenciadas para satisfacer la condición de estacionareidad de las series que es requerida para la estimación de un VAR. Asimismo, se realizó una prueba de cointegración entre el IVPI y el crecimiento del INPC para determinar la conveniencia de un VAR o un modelo de corrección de error (MCE) misma que, como era de esperarse, fue rechazada. ${ }^{37}$ El VAR en forma reducida incluyo 14 rezagos, una constante y variables binarias estacionales.

Un problema de la estimación empírica de la inflación latente es que la diferenciación realizada para satisfacer el requerimiento de estacionalidad de la series sólo permite la obtención del cambio en la inflación latente. Para recuperar su nivel es necesario un supuesto adicional sobre una condición inicial de la inflación. En este trabajo se siguió la propuesta de Alvarez y Sebastián (1995) seleccionado como nivel inicial de la inflación latente a aquél que minimiza la suma de las desviaciones al cuadrado respecto al crecimiento porcentual del INPC. Este criterio es equivalente a suponer que las perturbaciones con efecto de largo plazo sobre el producto provienen de una distribución de

\footnotetext{
${ }^{35}$ En el apéndice 3 se presenta una descripción detallada del uso de la metodología VARE para el cálculo de la inflación latente.

${ }^{36}$ Se optó por el IVPI en lugar del PIB para contar con datos mensuales.

${ }^{37}$ No se anticipaba que las series estuvieran cointegradas ya que se trata de una variable nominal y otra real.
} 
probabilidad simétrica con media cero, esto es, que la esperanza de la inflación no-latente es igual a cero y por lo tanto la inflación latente fluctúa alrededor del crecimiento porcentual del INPC.

En la gráfica 6 se compara a la inflación latente con el crecimiento mensual del INPC. Como ahí se observa, de 1990 a principios de 1993 ambas medidas muestran una clara tendencia a la baja. El hecho de que la inflación latente sigue una tendencia a la baja permite concluir que la política monetaria, bajo la situación de demanda agregada y de oferta agregada de corto plazo que prevaleció, fue compatible con el abatimiento de la inflación. Por otra parte, el hecho de que en dicho periodo el crecimiento del INPC es menor a la inflación latente es indicativo de choques positivos al producto de largo plazo, los cuales son debidos probablemente a las reformas estructurales 0 a la entrada de capitales que tuvieron lugar en esos años.

\section{Gráfica 6 INFLACIÓN LATENTE}

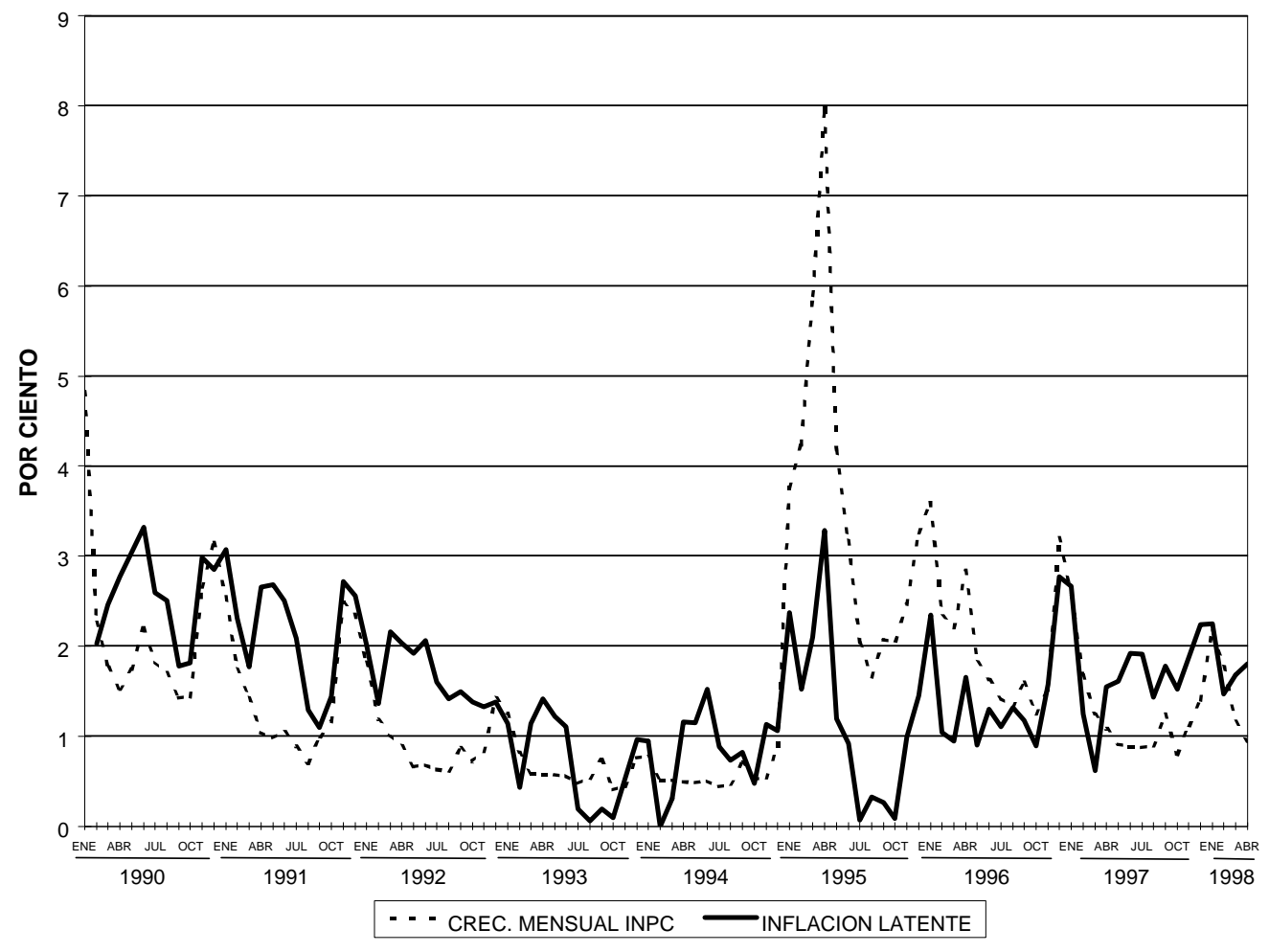

Durante 1993 y el primer trimestre de 1994 se observa que el efecto de los choques positivos al producto de largo plazo se diluye y la inflación latente fluctúa alrededor del crecimiento porcentual del INPC.

En el periodo que comprende del segundo trimestre de 1994 a finales de ese mismo año la inflación latente muestra una tendencia creciente mientras que el crecimiento del INPC permanece estable, este comportamiento es congruente con la existencia de perturbaciones positivas al producto de largo plazo y de presiones inflacionarias por el lado de la demanda agregada.

De enero a mayo de 1995 el crecimiento del INPC aumenta. La mayor parte de este crecimiento se puede atribuir al efecto que tuvo la crisis de diciembre de 1994 sobre el producto de largo plazo 
(debido posiblemente a salida de capitales, a la caída pronunciada de la inversión que tuvo que darse para cerrar el déficit en cuenta corriente, a la quiebra de empresas y al efecto del mecanismo de transmisión de la depreciación del tipo de cambio real, entre otros). La inflación latente también aumenta, probablemente por el efecto de la devaluación del tipo de cambio nominal y no por un relajamiento en la política monetaria.

A partir de junio de 1995 se observa una caída muy pronunciada de la inflación latente, este fenómeno pudo ser resultado de una política monetaria restrictiva o de una contracción de la demanda agregada por efecto de la crisis, por ejemplo, debido al menor consumo agregado como resultado de menores niveles de empleo. Cabe destacar que el hecho de que la brecha entre el crecimiento del INPC y la inflación latente se mantiene indica la existencia de nuevos choques negativos sobre el producto de largo plazo. Una posible fuente de estos choques es la restricción del crédito del sistema bancario posterior al inicio de la crisis que necesariamente afecta negativamente al producto de largo plazo.

De noviembre de 1995 a octubre de 1996 la inflación latente presenta una tendencia creciente lo cual se puede interpretar como resultado de la recuperación de la demanda agregada en un contexto de una política monetaria que satisface la demanda de medios de pago que dicha recuperación va requiriendo. Poco a poco la brecha entre el crecimiento del INPC y la inflación latente fue cerrándose, lo que indicaría que el efecto de los choques negativos al producto de largo plazo se fue diluyendo.

D esde el inicio de la crisis y hasta octubre de 1996, la mayor parte del crecimiento del INPC se debió a factores distintos de los cambios en la demanda agregada. A partir de diciembre de 1996 y hasta abril de 1998, la inflación latente sigue una tendencia creciente (con un incremento temporal significativo entre noviembre de 1996 y febrero de 1997) mientras que la tendencia del cambio porcentual del INPC (véase inflación subyacente) se ha estacionado en un mismo nivel. Este comportamiento de la inflación latente señala las dificultades que en este periodo enfrentó la política económica para abatir la inflación, dadas las condiciones de la demanda agregada y de la oferta agregada de corto plazo que prevalecieron. En particular, se observa que de mayo a diciembre de 1997 la inflación latente se situó encima del crecimiento del INPC lo que indicaría choques positivos al producto de largo plazo. Estos choques fueron resultado probablemente de las cuantiosas entradas de capitales y de la recuperación de la inversión, entre otros factores. De manera que la estabilidad del crecimiento del INPC observada en este periodo probablemente se vio favorecida por la presencia de choques positivos sobre el producto de largo plazo.

Finalmente, cabe destacar que durante el primer trimestre de 1998 la trayectoria de la inflación latente converge hacia el crecimiento del INPC, lo que hace suponer que en ausencia de nuevos choques positivos al producto de largo plazo, el abatimiento de la inflación requerirá de políticas económicas que reduzcan las presiones de demanda existentes, como serían la instrumentación de una política monetaria restrictiva o la contracción del gasto público.

\section{INFLACIÓN PERMANENTE}

Alvarez y Sebastián (1995) proponen la obtención de una medida de inflación de largo plazo utilizando la metodología del VAR estructural. El procedimiento estadístico es muy parecido al de la inflación latente, incluye las mismas variables (crecimiento porcentual de IPC y un indicador de producción real) y únicamente varía la restricción proveniente de la teoría económica. En este caso también se determinan dos tipo de perturbaciones, pero la identificación de las perturbaciones es con respecto a su efecto sobre la inflación Un tipo de perturbaciones tiene únicamente un impacto transitorio sobre la inflación mientras que el otro tipo genera cambios permanentes sobre esta variable. Estos autores 
mencionan que, bajo un supuesto de racionalidad, los cambios permanentes en la inflacón son los que se incorporan en la formación de expectativas. ${ }^{38}$

En este trabajo se ha argumentado que el crecimiento porcentual del IPC se encuentra contaminado por cambios en los precios relativos; la inflación permanente depura al IPC de estos cambios y de los movimientos temporales en el nivel general de precios. Por este motivo, la inflación permanente es un buen indicador del cambio sostenido en el nivel general de precios, ya que muestra el movimiento tendencial de la inflación sin eliminar información de los precios que componen al IPC.

La metodología para obtener la inflación permanente tampoco permite distinguir si los choques que la definen se originan en el lado de la oferta o de la demanda. Los cambios en la tasa de crecimiento monetario y los desplazamientos perdurables de la demanda de dinero son algunos cambios de demanda que generarían un efecto permanente sobre la tasa de inflacón Pero también choques de oferta de largo plazo o los cambios en la tasa de crecimiento de los salarios nominales generarían cambios en el componente permanente de la inflacón Por otro lado choques de oferta de corto plazo 0 cambios temporales de la política monetaria o fiscal generarían únicamente cambios transitorios en la inflación

Es posible obtener información sobre la permanencia de los choques que afectan el crecimiento de los precios al comparar a la inflación permanente con el crecimiento del IPC; cuando el cambio en el crecimiento del IPC corresponde en dirección y magnitud a un cambio en la inflación permanente se interpreta que el proceso inflacionario está influenciado por cambios permanentes. Esta información es valiosa ya que cualquier estrategia anti-inflacionaria debe enfocarse a contrarrestar el componente permanente del crecimiento de los precios. Cabe destacar que la información proporcionada por la inflación permanente es complementaria a la obtenida con la inflación latente y permite identificar el tipo de presiones inflacionarias presentes en un momento dado.

La inflación permanente se estimó para México con la misma muestra usada para la inflación latente. Cabe destacar que para recuperar su nivel, también es necesario hacer un supuesto adicional sobre la condición inicial de la inflación permanente. Sin embargo, en este caso, el supuesto de que la inflación permanente fluctúa alrededor del cambio porcentual del IPC tiene mucho más sustento ya que como se trata de una medida tendencial es lógico suponer que la esperanza de los cambios transitorios es cero y por lo tanto el promedio de las desviaciones de esta medida alrededor del IPC debe ser cero.

En la gráfica 7 se compara a la inflación permanente con el crecimiento mensual del INPC. Es posible separar el análisis en cuatro periodos de acuerdo al comportamiento de la inflación permanente con relación a los cambios en el crecimiento mensual del INPC. El primer periodo comprende los años 1990 y 1991, el segundo periodo comprende los años de 1992, 1993 y 1994, el tercer periodo va de enero de 1995 a mayo de 1996 y, finalmente, el cuarto periodo va de junio de 1996 a la fecha.

\footnotetext{
${ }^{38}$ En el apéndice 3 se describen con detalle las modificaciones a la metodología de Q uah y Vahey que permiten la obtención de la inflación permanente.
} 


\section{Gráfica 7 \\ INFLACIÓN PERMANENTE}

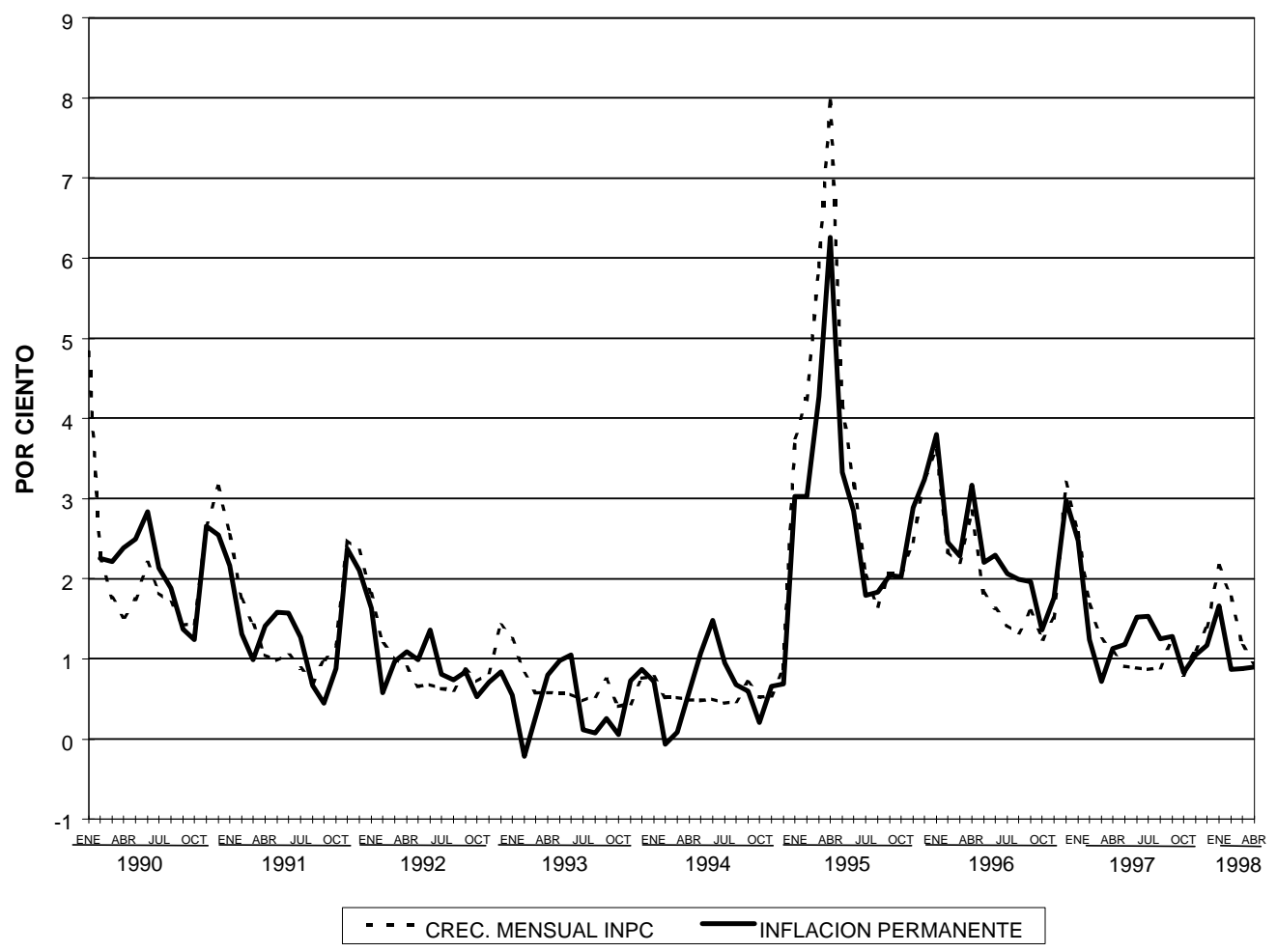

D urante el primer periodo los cambios del crecimiento mensual del INPC y los cambios de la inflación permanente son muy similares, ambos siguen una tendencia decreciente, por lo que se concluye que en esa época la reducción lograda de la inflación correspondió a una reducción de la inflación de largo plazo. En el segundo periodo, los movimientos de las dos medidas se disocian; el crecimiento del INPC se mantiene en un nivel estable mientras que la inflación permanente fluctúa alrededor de dicho nivel. Este comportamiento es indicativo de la presencia de choques permanentes sobre la inflación contrarrestados posiblemente por política económica de corto plazo.

Como se puede observar en la gráfica 7, en el tercer periodo los efectos de la crisis de diciembre de 1994 significaron cambios permanentes en la inflación, ya que en esa época los movimientos del crecimiento mensual del INPC correspondieron casi en su totalidad a cambios en la inflación permanente. Este resultado es congruente con el obtenido para la inflación latente, debido a que cambios en el producto de largo plazo no tienen un efecto sobre la inflación latente pero si generan cambios permanentes en la inflación. Finalmente, en el último periodo, los cambios en el crecimiento del INPC otra vez comienzan a disociarse de la inflación permanente lo que sería indicativo de que algunas reducciones del crecimiento del INPC a mediados de 1996 y 1997 se deben a factores con un efecto transitorio sobre la inflación.

\section{EVALUACION COMPARATIVA}

La búsqueda de medidas alternativas de inflación, como las presentadas en la exposición anterior, ha sido motivada por las deficiencias del IPC para captar el incremento sostenido en el nivel general de precios. Estas deficiencias también han oscurecido la evaluación empírica de la relación entre dinero y 
precios. Los cambios estacionales de precios y las variaciones de precios relativos no tienen un origen monetario y sólo constituyen un sesgo del IPC como estimador de la inflación. Por otro lado, como se discutió en la sección dedicada a la inflación latente, pueden existir presiones inflacionarias que sean ajenas al control de los agregados monetarios, como aquéllas debidas a cambios en el producto de largo plazo.

En esta sección se evalúa empíricamente para el caso de México la relación entre los diferentes indicadores de inflación y los agregados monetarios. Se esperaría que las medidas presentadas tuvieran una mejor relación con los agregados monetarios, especialmente los más estrechos (billetes y monedas en circulación y M1), que el crecimiento porcentual del INPC. Para evaluar esta relación entre dinero y precios se realizan dos pruebas. En primer lugar, como teóricamente se esperaría que el crecimiento de la cantidad de dinero determinara a la inflación, se realiza una prueba de causalidad estadística. ${ }^{39}$ En segundo lugar, se evalúa el poder explicativo del crecimiento en la cantidad de dinero para el pronóstico de la inflación.

La causalidad entre dinero y precios se evaluó mediante pruebas de causalidad estadística de Granger. Es decir, se determina si los cambios en el crecimiento de los agregados monetarios preceden a los cambios en la tasa de inflación, una vez que se ha considerado la capacidad histórica de la inflación para predecirse a si misma. Las pruebas se realizaron para el crecimiento porcentual del INPC y cada una de las medidas incluidas en este documento con los agregados monetarios billetes y monedas en circulación (ByM), M1, M2 y M4, respectivamente. La muestra utilizada fue de enero de 1990 a abril de 1998. Las pruebas se realizaron utilizando doce rezagos, es decir, los cambios en la inflación pueden verse influidos por su propia trayectoria y por el crecimiento monetario en el último año. En el cuadro 1, se presentan los resultados de estas pruebas al 5 y 10 por ciento de significancia.

\section{PRUEBAS DE CAUSALIDAD DE GRANGER}

\begin{tabular}{|c|c|c|c|c|c|c|c|c|}
\hline INFLACION & \multicolumn{2}{|c|}{$\begin{array}{l}\text { ByM } \\
\text { ES }\end{array}$} & \multicolumn{2}{|r|}{$\begin{array}{ll}\text { M1 } & \\
& \text { ES }\end{array}$} & \multicolumn{2}{|r|}{$\begin{array}{ll}\text { M2 } & \\
& \text { ES }\end{array}$} & \multicolumn{2}{|c|}{$\begin{array}{ll}\text { M4 } & \\
& \text { ES }\end{array}$} \\
\hline INPC & NO & NO & NO & NO & NO & NO & $\mathrm{SI}^{\star \star}$ & NO \\
\hline SUBYACENTE (ARIMA) & NO & $\mathrm{SI}^{*}$ & NO & NO & NO & NO & $\mathrm{SI}^{\star *}$ & NO \\
\hline NUCLEO & $\mathbf{S} \mathbf{l}^{\star *}$ & NO & NO & NO & NO & NO & $\mathrm{SI}^{\star *}$ & NO \\
\hline MEDIANA & $\mathrm{SI}^{*}$ & NO & NO & NO & NO & NO & $\mathrm{SI}^{*}$ & NO \\
\hline MEDIA TRUNCADA & $\mathrm{SI}^{*}$ & NO & NO & NO & NO & NO & $\mathrm{SI}^{\star \star}$ & NO \\
\hline LATENTE & $\mathbf{S I}^{\star \star}$ & NO & NO & NO & $\mathbf{S I}^{\star \star}$ & NO & $S I^{\star \star}$ & NO \\
\hline PERMANENTE & $\mathbf{S} \mathbf{l}^{\star *}$ & NO & NO & NO & NO & NO & $\mathbf{S I}^{\star *}$ & NO \\
\hline
\end{tabular}

* al $10 \%$ de significancia

** al $5 \%$ de significancia

Como se aprecia en el cuadro 1, para la muestra considerada, cuando se utiliza el INPC la relación causal sólo es significativa para el agregado monetario M4. Todas las demás medidas de inflación, a excepción de la inflación subyacente, presentan la causalidad esperada de billetes y monedas a precios. Estos resultados avalan la hipótesis de que los cambios temporales, de precios relativos o los debidos a modificaciones del producto de largo plazo, que son capturados por el INPC obscurecen la relación entre los agregados monetarios y la inflación, y que una vez que dicho indicador es depurado de ellos la relación vuelve a ser significativa. Las relaciones de causalidad no son significativas en ningún

\footnotetext{
${ }^{39}$ Algunas autores han encontrado resultados contraintuitivos en la relación de causalidad entre agregados monetarios e inflación, véase Hoover (1991) para el caso de E.U. y Pérez López (1998) para el caso de México. Los resultados obtenidos por estos autores pueden ser reflejo de los problemas que tiene el IPC como indicador de inflación.
} 
sentido y para ninguna de las medidas cuando se utiliza el agregado monetario M1. Cuando se utiliza el agregado monetario M2, sólo la inflación latente presenta causalidad de dinero a precios. El agregado monetario amplio M4 es el único para el cual la causalidad de dinero a precios se comprueba para cualquier definición de inflación.

Es importante destacar que la inflación latente es la única medida para la cual la causalidad de dinero a precios se cumple para casi todos los agregados monetarios, mientras que la inflación permanente presenta la relación causal más significativa para billetes y monedas y M4.

Para evaluar el poder explicativo de los cambios en los agregados monetarios en el pronóstico de la diferentes medidas de inflación se realiza una prueba que consiste en comparar los estadísticos $\mathrm{R}^{2}$ de una serie de regresiones que relacionan al crecimiento pasado del dinero, medido a través de distintos agregados monetarios, con cada medida de inflación en un cierto periodo posterior. De manera que una mayor $\mathrm{R}^{2}$ representa una mayor relación entre la medida y el agregado monetario respectivo. Para que dicha comparación sea válida es necesario establecer una especificación común para todas las medidas. Asimismo, deben considerarse diferentes horizontes de pronóstico para tomar en cuenta que los cambios en la cantidad de dinero pueden afectar con rezagos a la inflación. Para evitar problemas de multicolinealidad del crecimiento monetario se utilizó una especificación con rezagos distribuidos del tipo de Almon. La forma funcional de las regresiones es la siguiente: ${ }^{40}$

$$
\frac{1}{\mathrm{~K}}\left(\ln \mathrm{p}_{\mathrm{t}+\mathrm{K}}-\ln \mathrm{p}_{\mathrm{t}}\right)=\alpha+\beta\left[\sum_{\mathrm{i}=0}^{12} \mathrm{w}_{\mathrm{i}} \Delta \ln \mathrm{M}_{\mathrm{t}-\mathrm{i}}\right]+\varepsilon_{\mathrm{t}}
$$

D onde, Kes el horizonte de pronóstico futuro de la inflación, para el cual se consideraron los periodos de uno, tres, seis, nueve, doce y veinticuatro meses; pes el índice de precios de la medida respectiva y $\mathrm{Mes}$ el agregado monetario. Al igual que para la prueba anterior, se consideraron los agregados ByM, M1, M2 y M4. Asimismo, el orden del polinomio considerado fue de doce rezagos. Los estadísticos $\mathrm{R}^{2}$ se presentan en el cuadro 2.

En el cuadro 2 se puede apreciar que las ecuaciones que usan el incremento porcentual del INPC presentan un ajuste mucho menor que la mayoría de las medidas alternativas de inflación. Como se menciono en las secciones anteriores, con excepción de la inflación latente, todas las medidas buscan obtener el proceso tendencial o permanente de la inflación. Por este motivo, en general, para estas medidas tendenciales la capacidad de pronóstico de las ecuaciones aumenta cuando se considera un horizonte más largo. En particular, cuando se considera el horizonte de pronóstico de dos años destacan los resultados de la inflación permanente.

\footnotetext{
${ }^{40}$ Los rezagos de Almon pueden no ser la mejor representación de la relación entre dinero y precios, sin embargo, esta especificación permite considerar de forma sencilla la dinámica de dicha relación para fines comparativos.
} 
CUADRO 2

$R^{2}$ DE LAS ECUACIONES DE PRONOSTICO DE LA INFLACION

\begin{tabular}{|ll|r|r|r|r|r|r|r|}
\hline & & INPC & $\begin{array}{c}\text { SUBYACENTE } \\
\text { ARIMA }\end{array}$ & $\begin{array}{c}\text { INFLACION } \\
\text { NUCLEO }\end{array}$ & MEDIANA & $\begin{array}{c}\text { MEDIA } \\
\text { TRUNCADA }\end{array}$ & $\begin{array}{c}\text { INFLACION } \\
\text { LATENTE }\end{array}$ & $\begin{array}{c}\text { INFLACION } \\
\text { PERMANENTE }\end{array}$ \\
\hline ByM & $\mathbf{K}=\mathbf{1}$ & 0.024 & 0.037 & 0.060 & 0.042 & 0.039 & 0.456 & 0.010 \\
& $\mathbf{K}=\mathbf{2}$ & 0.015 & 0.029 & 0.039 & 0.027 & 0.025 & 0.405 & 0.003 \\
& $\mathbf{K}=\mathbf{3}$ & 0.010 & 0.022 & 0.027 & 0.022 & 0.018 & 0.376 & 0.007 \\
& $\mathbf{K}=\mathbf{6}$ & 0.005 & 0.011 & 0.003 & 0.001 & 0.003 & 0.542 & 0.005 \\
& $\mathbf{K}=\mathbf{1 2}$ & 0.011 & 0.015 & 0.013 & 0.010 & 0.013 & 0.423 & 0.023 \\
& $\mathbf{K}=\mathbf{2 4}$ & 0.125 & 0.121 & 0.133 & 0.123 & 0.128 & 0.128 & 0.134 \\
\hline $\mathbf{M 1}$ & $\mathbf{K}=\mathbf{1}$ & 0.149 & 0.182 & 0.176 & 0.147 & 0.151 & 0.252 & 0.117 \\
& $\mathbf{K}=\mathbf{2}$ & 0.147 & 0.189 & 0.180 & 0.152 & 0.154 & 0.281 & 0.127 \\
& $\mathbf{K}=\mathbf{3}$ & 0.146 & 0.198 & 0.183 & 0.157 & 0.156 & 0.311 & 0.148 \\
& $\mathbf{K}=\mathbf{6}$ & 0.175 & 0.229 & 0.198 & 0.173 & 0.179 & 0.411 & 0.226 \\
& $\mathbf{K}=\mathbf{1 2}$ & 0.307 & 0.325 & 0.319 & 0.301 & 0.313 & 0.321 & 0.362 \\
& $\mathbf{K}=\mathbf{2 4}$ & 0.569 & 0.578 & 0.576 & 0.571 & 0.570 & 0.117 & 0.631 \\
\hline M2 & $\mathbf{K}=\mathbf{1}$ & 0.110 & 0.074 & 0.116 & 0.107 & 0.102 & 0.277 & 0.119 \\
& $\mathbf{K}=\mathbf{2}$ & 0.084 & 0.074 & 0.112 & 0.095 & 0.086 & 0.310 & 0.096 \\
& $\mathbf{K}=\mathbf{3}$ & 0.072 & 0.076 & 0.101 & 0.084 & 0.075 & 0.342 & 0.092 \\
& $\mathbf{K}=\mathbf{6}$ & 0.058 & 0.093 & 0.088 & 0.075 & 0.065 & 0.472 & 0.110 \\
& $\mathbf{K}=\mathbf{1 2}$ & 0.197 & 0.194 & 0.205 & 0.190 & 0.199 & 0.547 & 0.166 \\
& $\mathbf{K}=\mathbf{2 4}$ & 0.439 & 0.425 & 0.449 & 0.424 & 0.439 & 0.437 & 0.414 \\
\hline $\mathbf{M 4}$ & $\mathbf{K}=\mathbf{1}$ & 0.106 & 0.048 & 0.149 & 0.140 & 0.130 & 0.182 & 0.064 \\
& $\mathbf{K}=\mathbf{2}$ & 0.091 & 0.058 & 0.138 & 0.126 & 0.114 & 0.200 & 0.053 \\
& $\mathbf{K}=\mathbf{3}$ & 0.081 & 0.065 & 0.121 & 0.114 & 0.101 & 0.237 & 0.060 \\
& $\mathbf{K}=\mathbf{6}$ & 0.047 & 0.072 & 0.071 & 0.061 & 0.055 & 0.350 & 0.047 \\
& $\mathbf{K}=\mathbf{1 2}$ & 0.102 & 0.103 & 0.115 & 0.106 & 0.109 & 0.471 & 0.067 \\
$\mathbf{K}=\mathbf{2 4}$ & 0.138 & 0.138 & 0.148 & 0.138 & 0.143 & 0.399 & 0.149 \\
\hline
\end{tabular}

La inflación latente resalta como la medida cuya ecuación presenta, en general, el mejor ajuste, especialmente para el pronóstico de la inflación de corto y mediano plazo. Este resultado permite concluir que en México el crecimiento de los precios que se origina por perturbaciones con un efecto de largo plazo sobre el producto contamina la relación entre dinero y precios. Asimismo, cabe destacar que el mejor ajuste de la inflación latente se presenta con ByM.

Finalmente, de estas dos pruebas se puede concluir que en general todas las medidas alternativas presentan un mejoría respecto al INPC. La inflación latente y permanente son las que presentan las mejores relaciones de causalidad. Por su relación con los agregados monetarios destacan en el corto y mediano plazo la inflación latente y en el largo plazo como medida tendencia la inflación permanente.

\section{CONSIDERACIONES FINALES}

Para el estudio empírico de cualquier fenómeno económico es de particular relevancia contar con indicadores que reflejen fielmente el objeto de estudio. Esto es más importante mientras más amplio es el ámbito de influencia del fenómeno en cuestión. En particular, la inflación, por sus múltiples y variados efectos sobre la asignación de recursos, el crecimiento del producto y el bienestar de la población, ha sido objeto de estudio tanto teórico como empírico y ha acaparado la atención de renombrados economistas.

El concepto teórico de inflación se refiere al crecimiento sostenido del nivel general de precios. En la práctica, se ha utilizado al crecimiento porcentual del IPC como el indicador más importante de la inflación. Sin embargo, existen diversas consideraciones, tanto teóricas como empíricas, que cuestionan la efectividad del crecimiento porcentual del IPC como indicador del crecimiento sostenido de los precios. Por un lado, el IPC es un índice cuya motivación principal es medir el nivel de los 
precios relevantes para un consumidor típico y no el nivel general de los precios. Por otro lado, al limitarse a una muestra específica de precios, los movimientos del IPC reflejan cambios estacionales o de precios relativos y modificaciones en los precios debidas a cambios en la oferta agregada. De manera que existe una desconexión entre la medida que se utiliza comúnmente como indicador de inflación y su contraparte teórica.

En este documento se presentan una serie de medidas alternativas de inflación cuya finalidad es subsanar las deficiencias del cambio porcentual del IPC. Los estimadores considerados fueron: la inflación subyacente, el núcleo inflacionario, las medidas de inflación con estimadores de influencia limitada (mediana y media truncada), la inflación permanente y la inflación latente. Los primeros cuatro tienen como finalidad obtener la tendencia del crecimiento del IPC, ya sea como resultado de metodologías estadísticas (inflación subyacente), como de la construcción de un nuevo índice de precios que excluye algunos de los bienes y servicios que forman la canasta del IPC (núcleo, mediana o media truncada), o como los cambios en la inflación que perduran en el largo plazo (inflación permanente). En estos casos se interpreta que la inflación es el movimiento sostenido en el nivel general de precios y es de esperarse que los precios de los bienes que conforman la canasta del IPC, una vez depurados de las fluctuaciones de corto plazo mediante la obtención de la tendencia, varíen simultáneamente con el nivel general de los precios.

La inflación latente, en cambio, sigue un enfoque distinto: utiliza algunas de las características teóricas distintivas de la inflación (en particular, que la inflación es el cambio en magnitudes nominales que en el largo plazo no tienen efectos reales) para identificarla a partir de información empírica. Este enfoque tiene la ventaja que la medida obtenida tiene una interpretación directamente relacionada con la teoría económica. La inflación latente se define como el crecimiento de los precios que existiría en ausencia de choques al producto de largo plazo. En particular, se interpreta como la medida que responde a modificaciones de la demanda agregada y de la oferta agregada de corto plazo, sobre la cual se esperaría que la política monetaria tuviera cierta influencia.

Estas medidas son utilizadas por diversos países, ya sea individualmente 0 alguna combinación de ellas para el seguimiento del fenómeno inflacionario. En este trabajo se estiman para México y, en términos generales, se puede decir que cada una ofrece información sobre el proceso inflacionario. Sin embargo, algunas de estas medidas proveen información similar y difieren en cuanto a su complejidad técnica.

La evaluación empírica de estas medidas señala que la inflación latente es un buen indicador de inflación de corto y mediano plazo, ya que presenta una causalidad estadística de dinero a precios y una relación significativa con la mayoría de los agregados monetarios, además de que permite un análisis exhaustivo de las presiones inflacionarias. Por otro lado, de los resultados de las pruebas estadísticas se puede concluir que la inflación permanente es un buen indicador tendencial de la inflación. Si bien estas dos medidas parecen ser candidatos adecuados como indicadores del proceso inflacionario en México, sería recomendable continuar el análisis de estas medidas alternativas de inflación. De esta forma se podrá identificar cuál o cuáles medidas son las de mayor utilidad, de manera que tanto autoridades como analistas cuenten con mejores indicadores sobre este fenómeno económico. 


\section{APÉN DICE 1}

BIENES Y SERVICIOS DEL INPC QUE SE EXCLUYEN EN LA ELABORACIÓN DE LA INFLACIÓN NÚCLEO

(Ordenados de mayor a menor volatilidad)

\begin{tabular}{|c|c|c|c|c|c|}
\hline ELEMENTO & $\begin{array}{l}\text { PORCENTAJE } \\
\text { DEL INGRESO } \\
\text { ACUMULADO }\end{array}$ & ELEMENTO & $\begin{array}{l}\text { PORCENTAJE } \\
\text { DEL INGRESO } \\
\text { ACUMULADO }\end{array}$ & ELEMENTO & $\begin{array}{l}\text { PORCENTAJE } \\
\text { DEL INGRESO } \\
\text { ACUMULADO }\end{array}$ \\
\hline CHILE POBLANO & $1 \%$ & LECHUGA & $12 \%$ & CHILE SECO & $36 \%$ \\
\hline TOMATE VERDE & $1 \%$ & LARGA DISTANCIA INTERNACIO & $13 \%$ & CUOTAS DE AUTOPISTAS & $36 \%$ \\
\hline CHILE SERRANO & $1 \%$ & GASOLINA & $16 \%$ & LECHE MATERNIZADA & $36 \%$ \\
\hline METRO O TRANSP. ELECTRICO & $1 \%$ & ELECTRICIDAD & $18 \%$ & LECHE PASTEURIZADA ENV. & $37 \%$ \\
\hline LIMON & $1 \%$ & LARGA DISTANCIA NACIONAL & $19 \%$ & CAFE TOSTADO & $38 \%$ \\
\hline CALABACITA & $2 \%$ & PLATANO TABASCO & $19 \%$ & OTRAS PRENDAS P/HOMBRE & $38 \%$ \\
\hline CEBOLLA & $2 \%$ & SERVICIO TELEFONICO LOCAL & $20 \%$ & MOJARRA & $38 \%$ \\
\hline JITOMATE & $3 \%$ & AZUCAR & $20 \%$ & POLLO ENTERO & $38 \%$ \\
\hline OTROS CHILES FRESCOS & $3 \%$ & LINEA TELEFONICA & $20 \%$ & HOSPITALIZACION & $38 \%$ \\
\hline NOPALES & $3 \%$ & AUTOBUS URBANO & $22 \%$ & PAN DE CAJA & $39 \%$ \\
\hline EJOTES & $3 \%$ & MANZANA & $22 \%$ & ROBALO Y MERO & $39 \%$ \\
\hline OTRAS LEGUMBRES & $3 \%$ & HARINAS DE TRIGO & $22 \%$ & ATUN EN LATA & $39 \%$ \\
\hline OTROS CONDIMENTOS & $3 \%$ & OTROS PLATANOS & $22 \%$ & ANTICONCEPTIVOS Y HORMONALES & $39 \%$ \\
\hline OTROS ALIMENTOS COCINADOS & $3 \%$ & FERROCARRIL & $23 \%$ & SERVICIO DE BAÑO & $39 \%$ \\
\hline CHICHARO & $3 \%$ & ELOTE & $23 \%$ & LECHE SIN ENVASAR & $40 \%$ \\
\hline CHAYOTE & $3 \%$ & DURAZNO & $23 \%$ & TAXI & $40 \%$ \\
\hline TENENCIA DE AUTOMOVIL & $4 \%$ & CLUB DEPORTIVO & $23 \%$ & OTROS PESCADOS & $40 \%$ \\
\hline AGUACATE & $4 \%$ & PERA & $23 \%$ & ANALISIS & $41 \%$ \\
\hline PEPINO & $4 \%$ & ESTACIONAMIENTO & $23 \%$ & OTROS MARISCOS & $41 \%$ \\
\hline PAPAYA & $4 \%$ & PAN BLANCO & $24 \%$ & HOTELES & $41 \%$ \\
\hline NARANJA & $4 \%$ & SEGURO DE AUTOMOVIL & $24 \%$ & HUACHINANGO & $41 \%$ \\
\hline MANGO & $4 \%$ & TORTILLA DE MAIZ & $26 \%$ & UNIFORME PARA NIÑA & $41 \%$ \\
\hline MELON & $5 \%$ & CIGARRILLOS & $26 \%$ & PIERNA & $41 \%$ \\
\hline ZANAHORIA & $5 \%$ & UNIVERSIDAD & $27 \%$ & CUIDADO DENTAL & $42 \%$ \\
\hline GUAYABA & $5 \%$ & MANTECA VEGETAL & $27 \%$ & TRANSPORTE AEREO & $42 \%$ \\
\hline PAPA & $5 \%$ & PERIODICOS & $27 \%$ & LOMO & $42 \%$ \\
\hline TORONJA & $5 \%$ & ACEITE VEGETAL & $28 \%$ & JUGUETES & $42 \%$ \\
\hline JARDIN DE NIÑOS Y GUARDERIA & $5 \%$ & HARINA DE MAIZ & $28 \%$ & OTRAS LEGUMBRES SECAS & $42 \%$ \\
\hline SANDIA & $5 \%$ & LECHE EVAPORADA & $28 \%$ & SARDINA EN LATA & $42 \%$ \\
\hline AJO & $6 \%$ & CERVEZA & $29 \%$ & FOCOS & $42 \%$ \\
\hline UVA & $6 \%$ & JOYAS Y BISUTERIA & $29 \%$ & FRUTAS Y LEG.PREP.P/BEBES & $42 \%$ \\
\hline PRIMARIA & $6 \%$ & PETROLEO DIAFANO & $29 \%$ & OTROS PESC.Y MARIS.EN CONSERVA & $43 \%$ \\
\hline SECUNDARIA & $7 \%$ & HUEVO & $30 \%$ & ACEITES LUBRICANTES & $43 \%$ \\
\hline CUOTAS LIC.Y O.DOCUMENTOS & $7 \%$ & FRIJOL & $31 \%$ & SUETER PARA NIÑO & $43 \%$ \\
\hline COLECTIVO & $9 \%$ & POLLO EN PIEZAS & $32 \%$ & CAMARON & $43 \%$ \\
\hline PIÑA & $9 \%$ & LECHE EN POLVO & $32 \%$ & BLANQUEADORES Y LIMPIADORES & $43 \%$ \\
\hline $\mathrm{COL}$ & $9 \%$ & LECHE CONDENSADA & $32 \%$ & SERV. DE TINT. Y LAVANDERIA & $44 \%$ \\
\hline CARRERA CORTA E IDIOMAS & $10 \%$ & CAFE SOLUBLE & $32 \%$ & PULPA DE CERDO & $44 \%$ \\
\hline GAS DOMESTICO & $11 \%$ & MASA DE MAIZ & $33 \%$ & ARROZ & $44 \%$ \\
\hline PREPARATORIA & $12 \%$ & AUTOBUS FORANEO & $34 \%$ & PAÑALES & $44 \%$ \\
\hline ESPECTACULOS DEPORTIVOS & $12 \%$ & REFRESCOS ENVASADOS & $36 \%$ & PURE DE TOMATE & $44 \%$ \\
\hline
\end{tabular}




\section{APÉNDICE 2}

Frecuencia con que cada Componente del INPC queda excluido de la MediaTruncada (Ordenados de Mayor a Menor)

\begin{tabular}{|c|c|c|c|c|c|}
\hline ELEMENTO & FRECUENCIA* & ELEMENTO & FRECUENCIA* & ELEMENTO & FRECUENCIA* \\
\hline CHILE POBLANO & 88.3 & CIGARRILLOS & 22.2 & CLUB DEPORTIVO & 11.1 \\
\hline TOMATE VERDE & 86.5 & JARDIN DE NIÑOS Y GUARDERIA & 21.6 & AUTOBUS FORANEO & 11.1 \\
\hline CALABACITA & 85.4 & ACEITE VEGETAL & 19.9 & ESTACIONAMIENTO & 11.1 \\
\hline CHILE SERRANO & 84.2 & MANTECA VEGETAL & 19.9 & PAN BLANCO & 10.5 \\
\hline JITOMATE & 83.0 & METRO O TRANSP. ELECTRICO & 19.9 & LECHE EN POLVO & 10.5 \\
\hline EJOTES & 82.5 & FERROCARRIL & 19.9 & MANTECA DE CERDO & 10.5 \\
\hline LIMON & 81.9 & HARINAS DE TRIGO & 19.3 & MERMELADAS & 10.5 \\
\hline OTROS CHILES FRESCOS & 81.3 & FRIJOL & 19.3 & CORTINAS & 10.5 \\
\hline CEBOLLA & 80.7 & OTRAS LEGUMBRES SECAS & 19.3 & OTROS LICORES & 9.9 \\
\hline CHICHARO & 78.9 & PAÑUELOS DESECHABLES & 18.7 & NEUMATICOS & 9.9 \\
\hline NOPALES & 78.9 & YOGURT & 18.1 & ATUN EN LATA & 9.4 \\
\hline CHAYOTE & 78.4 & PRIMARIA & 18.1 & CONCENTRADO DE POLLO & 9.4 \\
\hline PEPINO & 77.8 & PAPEL HIGIENICO & 17.5 & CAJETAS & 9.4 \\
\hline NARANJA & 72.5 & SECUNDARIA & 17.5 & JUGOS O NECTARES ENVASADOS & 9.4 \\
\hline AGUACATE & 71.3 & SEGURO DE AUTOMOVIL & 17.5 & VESTIDO PARA NIÑA & 9.4 \\
\hline OTRAS LEGUMBRES & 71.3 & LARGA DISTANCIA INTERNACIO & 17.5 & SUETER PARA NIÑA & 9.4 \\
\hline PAPAYA & 70.8 & HARINA DE MAIZ & 17.0 & ANTIGRIPALES & 9.4 \\
\hline SANDIA & 70.8 & LARGA DISTANCIA NACIONAL & 17.0 & ANTICONCEPTIVOS Y HORMONALES & 9.4 \\
\hline ZANAHORIA & 69.6 & LECHE CONDENSADA & 16.4 & MATERIAL Y APARATOS FOTOGRAFICOS & 9.4 \\
\hline TORONJA & 69.0 & COLCHONES & 16.4 & PERIODICOS & 9.4 \\
\hline LECHUGA & 67.3 & CUOTAS DE AUTOPISTAS & 16.4 & CUOTAS LIC.Y O.DOCUMENTOS & 9.4 \\
\hline PIÑA & 66.7 & SALCHICHAS & 15.8 & PASTELILLOS Y PASTELES & 8.8 \\
\hline MELON & 66.1 & SERVILLETAS DE PAPEL & 15.8 & PULPA DE CERDO & 8.8 \\
\hline OTROS CONDIMENTOS & 65.5 & TRAJES & 15.8 & CHULETA & 8.8 \\
\hline $\mathrm{COL}$ & 63.2 & TRANSPORTE AEREO & 15.8 & OTROS QUESOS & 8.8 \\
\hline OTROS ALIMENTOS COCINADOS & 62.6 & MEDIAS Y PANTIMEDIAS & 15.2 & MAYONESA & 8.8 \\
\hline PAPA & 62.0 & PREPARATORIA & 15.2 & FRUTAS Y LEG.PREP.P/BEBES & 8.8 \\
\hline AJO & 62.0 & LINEA TELEFONICA & 15.2 & RON & 8.8 \\
\hline PLATANO TABASCO & 57.3 & SERVICIO TELEFONICO LOCAL & 15.2 & FOCOS & 8.8 \\
\hline OTROS PLATANOS & 57.3 & JAMON & 14.6 & ROPA INTERIOR PARA NIÑA & 8.8 \\
\hline GUAYABA & 52.6 & CAFE TOSTADO & 14.6 & RECAMARAS & 8.8 \\
\hline JOYAS Y BISUTERIA & 50.9 & CERVEZA & 14.6 & COLCHAS & 8.8 \\
\hline DURAZNO & 47.4 & TOALLAS SANITARIAS & 14.6 & INSTS. MUSICALES Y OTROS & 8.8 \\
\hline MANGO & 46.2 & ABRIGOS & 14.6 & ESPECTACULOS DEPORTIVOS & 8.8 \\
\hline ELOTE & 45.6 & CARRERA CORTA E IDIOMAS & 14.6 & TORTILLA DE MAIZ & 8.2 \\
\hline PERA & 44.4 & JUGUETES & 14.6 & OTRAS GALLETAS & 8.2 \\
\hline UVA & 43.9 & LECHE EVAPORADA & 14.0 & PIERNA & 8.2 \\
\hline MANZANA & 42.7 & BLANQUEADORES Y LIMPIADORES & 14.0 & SARDINA EN LATA & 8.2 \\
\hline CHILE SECO & 35.7 & TOCINO & 13.5 & MARGARINA & 8.2 \\
\hline OTROS PESCADOS & 32.7 & OTROS EMBUTIDOS & 13.5 & OTRAS CONSERVAS DE FRUTAS & 8.2 \\
\hline MOJARRA & 32.7 & CAFE SOLUBLE & 13.5 & PAPAS FRITAS Y SIMILARES & 8.2 \\
\hline POLLO EN PIEZAS & 32.2 & PAN DE CAJA & 12.9 & PANTALON HOMBRE O/MATERIALES & 8.2 \\
\hline ROBALO Y MERO & 31.6 & QUESO CHIHUAHUA O MANCHEGO & 12.3 & CAMISAS & 8.2 \\
\hline HUEVO & 29.2 & SUETER PARA NIÑO & 12.3 & ROPA INTERIOR PARA NIÑO & 8.2 \\
\hline ELECTRICIDAD & 27.5 & GELATINA EN POLVO & 11.7 & BOLSAS, MALETAS Y CINTURON & 8.2 \\
\hline CAMARON & 26.9 & TEQUILA & 11.7 & BATERIAS DE COCINA & 8.2 \\
\hline TENENCIA DE AUTOMOVIL & 26.3 & CREMAS PARA LA PIEL & 11.7 & MUEBLES P/COCINA & 8.2 \\
\hline HUACHINANGO & 25.7 & PAÑALES & 11.7 & ANTIBIOTICOS & 8.2 \\
\hline OTROS MARISCOS & 25.1 & CHAMARRAS & 11.7 & ANALISIS & 8.2 \\
\hline UNIVERSIDAD & 24.6 & PASTA PARA SOPA & 11.1 & PLUMAS, LAPICES Y OTROS & 8.2 \\
\hline POLLO ENTERO & 23.4 & PASTEL DE CARNE & 11.1 & REVISTAS & 8.2 \\
\hline AZUCAR & 23.4 & VINO DE MESA & 11.1 & GALLETAS POPULARES & 7.6 \\
\hline
\end{tabular}

*/ Porcentaje del total de observaciones en la muestra. 
Frecuencia con que cada Componente del INPC queda excluido de la MediaTruncada (Ordenados de Mayor a Menor)

\begin{tabular}{|c|c|c|c|c|c|}
\hline ELEMENTO & FRECUENCIA* & ELEMENTO & FRECUENCIA* & ELEMENTO & FRECUENCIA* \\
\hline ARROZ & 7.6 & EXPECTORANTES Y DESCONGESTIVOS & 5.8 & COLECTIVO & 3.5 \\
\hline OTRAS VISCERAS DE RES & 7.6 & ARTICULOS DEPORTIVOS & 5.8 & MIEL DE ABEJA & 2.9 \\
\hline LOMO & 7.6 & CINE & 5.8 & DULCES Y CARAMELOS & 2.9 \\
\hline LECHE MATERNIZADA & 7.6 & MANTENIMIENTO DE AUTOMOVIL & 5.8 & PASTA DENTAL & 2.9 \\
\hline SAL & 7.6 & HIGADO DE RES & 5.3 & PANTALON HOMBRE B/ALGODON & 2.9 \\
\hline PRODUCTOS P/EL CABELLO & 7.6 & LECHE PASTEURIZADA ENV. & 5.3 & CALZONCILLOS & 2.9 \\
\hline SERVICIO DE BAÑO & 7.6 & QUESO AMARILLO & 5.3 & BLUSA P/NIÑOS & 2.9 \\
\hline CALCETINES & 7.6 & UTEN.DE PLAST. P/EL HOGAR & 5.3 & ZAPATOS P/MUJER & 2.9 \\
\hline OTRAS PRENDAS P/HOMBRE & 7.6 & VESTIDO PARA MUJER & 5.3 & ZAPATOS TENIS & 2.9 \\
\hline RELOJES & 7.6 & PANTALON MUJER O/MATERIALES & 5.3 & CALENTADORES PARA AGUA & 2.9 \\
\hline SABANAS & 7.6 & OTRAS PRENDAS P/MUJER & 5.3 & OTROS ARTICULOS DE TOCADOR & 2.9 \\
\hline CUADERNOS Y CARPETAS & 7.6 & LOZA Y CRISTALERIA & 5.3 & RADIOS Y GRABADORAS & 2.9 \\
\hline MASA DE MAIZ & 7.0 & PLANCHAS ELECTRICAS & 5.3 & TAXI & 2.9 \\
\hline CARNES AHUMADAS O ENCHILADAS & 7.0 & OTROS MEDICAMENTOS & 5.3 & AUTOBUS URBANO & 2.9 \\
\hline MANTEQUILLA & 7.0 & LIBROS DE TEXTO & 5.3 & OTRAS REFACCIONES & 2.9 \\
\hline CHILES PROCESADOS & 7.0 & SERV. FUNERARIOS & 5.3 & CREMA DE LECHE & 2.3 \\
\hline REFRESCOS ENVASADOS & 7.0 & LECHE SIN ENVASAR & 4.7 & HELADOS & 2.3 \\
\hline PANTALON NIÑO O/MATERIALES & 7.0 & QUESO FRESCO & 4.7 & JABON PARA LAVAR & 2.3 \\
\hline ROPA INTERIOR PARA MUJER & 7.0 & CHOCOLATE EN POLVO & 4.7 & JABON DE TOCADOR & 2.3 \\
\hline UNIFORME PARA NIÑA & 7.0 & PIMIENTA & 4.7 & ZAPATOS P/HOMBRE & 2.3 \\
\hline COMEDORES & 7.0 & MOSTAZA & 4.7 & REPARACION DE CALZADO & 2.3 \\
\hline NUTRICIONALES & 7.0 & LOCIONES Y PERFUMES & 4.7 & SOMBREROS & 2.3 \\
\hline GASTROINTESTINALES & 7.0 & SALA DE BELLEZA & 4.7 & REFRIGERADORES & 2.3 \\
\hline CUIDADO DENTAL & 7.0 & ZAPATOS P/NIÑOS & 4.7 & LENTES Y OTROS APARAT & 2.3 \\
\hline GASOLINA & 7.0 & LAVADORAS DE ROPA & 4.7 & TELEVISORES Y VIDEOCASETERAS & 2.3 \\
\hline ACEITES LUBRICANTES & 7.0 & TOALLAS & 4.7 & MANTENIMIENTO DE VIVIENDA & 2.3 \\
\hline ACUMULADORES & 7.0 & HOSPITALIZACION & 4.7 & RETAZO & 1.8 \\
\hline MAIZ & 6.4 & CARDIOVASCULARES & 4.7 & BARBACOA O BIRRIA & 1.8 \\
\hline CHORIZO & 6.4 & OTRAS DIVERSIONES & 4.7 & CAFETERIAS & 1.8 \\
\hline PURE DE TOMATE & 6.4 & OTROS PESC.Y MARIS.EN CONSERVA & 4.1 & CORTE DE CABELLO & 1.8 \\
\hline SOPAS ENLATADAS & 6.4 & QUESO OAXACA O ASADERO & 4.1 & OPERACION QUIR. Y PARTOS & 1.8 \\
\hline CHOCOLATE EN TABLETA & 6.4 & HOTELES & 4.1 & OTROS LIBROS & 1.8 \\
\hline BRANDY & 6.4 & DESODORANTES AMBIENTALES & 4.1 & OTROS UTENSILIOS DE COCINA & 1.8 \\
\hline NAVAJAS Y MAQ.DE AFEITAR & 6.4 & CERILLOS & 4.1 & PAN DULCE & 1.2 \\
\hline CONJUNTO PARA MUJER & 6.4 & BLUSA PARA MUJER & 4.1 & CORTES ESPECIALES DE RES & 1.2 \\
\hline ANTECOMEDORES & 6.4 & TRAJE P/BEBE & 4.1 & CARNITAS & 1.2 \\
\hline HILOS Y ESTAMBRES & 6.4 & CAMISETA P/BEBE & 4.1 & DETERGENTES & 1.2 \\
\hline DISCOS Y CASETES & 6.4 & ESTUFAS & 4.1 & CENTRO NOCTURNO & 1.2 \\
\hline MATERIAL DE CURACION & 6.4 & LICUADORAS & 4.1 & BICICLETAS & 1.2 \\
\hline GAS DOMESTICO & 6.4 & SALAS & 4.1 & CARNES SECAS & 0.6 \\
\hline PETROLEO DIAFANO & 6.4 & COBIJAS & 4.1 & LONCHERIAS & 0.6 \\
\hline FECULA DE MAIZ & 5.8 & CONSULTA MEDICA & 4.1 & HUARACHES Y SANDALIAS & 0.6 \\
\hline CEREALES EN HOJUELA & 5.8 & OTROS BLANCOS P/EL HOGAR & 4.1 & VENTILADORES & 0.6 \\
\hline VERDURAS ENVASADAS & 5.8 & ESCOBAS & 3.5 & OTROS APARATOS ELECTRICOS & 0.6 \\
\hline CONCEN PARA REFRESCOS & 5.8 & SERV. DE TINT. Y LAVANDERIA & 3.5 & PILAS & 0.6 \\
\hline POLLOS ROSTIZADOS & 5.8 & CAMISETAS & 3.5 & BISTEC DE RES & 0.0 \\
\hline DESODORANTES PERSONALES & 5.8 & PANTALON P/NIÑO B/ALGODON & 3.5 & CARNE MOLIDA DE RES & 0.0 \\
\hline ARTICULOS DE MAQUILLAJE & 5.8 & PANTALON MUJER B/ALGODON & 3.5 & CANTINAS & 0.0 \\
\hline UNIFORME PARA NIÑO & 5.8 & CALCETINES Y CALCETAS & 3.5 & RESTAURANTES & 0.0 \\
\hline FALDA PARA MUJER & 5.8 & EQUIPOS MODULARES & 3.5 & SERVICIO DOMESTICO & 0.0 \\
\hline MAQUINAS DE COSER & 5.8 & AUTOMOVILES & 3.5 & PLAGUICIDAS & 0.0 \\
\hline \multirow[t]{2}{*}{ ANALGESICOS } & 5.8 & VELAS Y VELADORAS & 3.5 & VIVIENDA PROPIA & 0.0 \\
\hline & & & & RENTA DE VIVIENDA & 0.0 \\
\hline
\end{tabular}

*/ Porcentaje del total de observaciones en la muestra. 


\section{APÉNDICE 3 \\ DESCRIPCIÓN DE LA METODOLOGÍA PARA EL CALCULO DE LAS INFLACIONES LATENTE Y PERMANENTE}

\section{INFLACIÓN LATENTE}

La estimación de la inflación latente está basada en el uso de un vector autorregresivo estructural (VARE) con la metodología propuesta por Blanchard y Q uah (1989). El uso de esta metodología para el cálculo de la inflación latente fue propuesto por Q uah y Vahey (1993).

Esta medida descompone al crecimiento porcentual del IPC identificando dos tipos de perturbaciones estructurales $\left(\varepsilon_{1}\right.$ y $\left.\varepsilon_{2}\right)$, que afectan al producto y a la inflación. El primer tipo de perturbaciones $\varepsilon_{1}$ agrupa a aquellos choques cuyo efecto sobre el producto desaparece del mediano al largo plazo, los cuales definen a la inflación latente. El segundo tipo de perturbaciones incluye a aquellos choques que tienen un efecto de largo plazo sobre la producción.

Sin embargo, las perturbaciones estructurales no son directamente observables y es necesario identificarlas. Para ello se requiere descomponer las series observables del crecimiento porcentual del IPC y de algún indicador de actividad económica real. O tra característica distintiva de esta metodología es que incorpora restricciones provenientes de la teoría económica. En particular, la misma definición de la inflación latente se impone como una restricción estadística, es decir, un tipo de perturbaciones no tendrá un efecto permanente sobre la actividad económica.

Si se define al logaritmo del producto y la inflación como Y y $\Pi$ respectivamente y se supone que estas variables son integradas de orden uno y que no existe una relación de cointegración entre ellas, el VARE, en forma vectorial, puede escribirse de la siguiente manera:

donde:

$$
\begin{aligned}
\mathrm{X}_{\mathrm{t}} & =\mathrm{C}(0) \varepsilon_{\mathrm{t}}+\mathrm{C}(1) \varepsilon_{\mathrm{t}-1}+\mathrm{C}(2) \varepsilon_{\mathrm{t}-2}+\ldots \\
& =\mathrm{C}(\mathrm{L}) \varepsilon_{\mathrm{t}}
\end{aligned}
$$

$\mathrm{X}_{\mathrm{t}}=\left(\Delta \mathrm{Y}_{\mathrm{t}}, \Delta \Pi_{\mathrm{t}}\right)^{\prime}$

$\varepsilon_{\mathrm{t}}=\left(\varepsilon_{1_{\mathrm{t}}}, \varepsilon_{2 \mathrm{t}}\right)^{\prime}$

$L \quad$ es el operador de rezagos,

$\Delta \quad$ el operador de diferencias $(\Delta=(1-\mathrm{L}))$,

$C(L)$ es una matriz $2 \mathrm{x} 2$ de polinomios de rezagos cuyos coeficientes determinan los mecanismos de transmisión de las perturbaciones estructurales.

La ecuación (1) supone que el cambio en la inflación y el producto puede ser representado como una distribución de rezagos de las perturbaciones estructurales. De esta forma, el efecto sobre $\mathrm{x}_{\mathrm{t}}$ de las perturbaciones contemporáneas esta dado por la submatriz $C(0)$ y el efecto de los choques pasados por $C(k), k \geq 1$.

En términos de los mecanismos de transmisión, para que la perturbación $\varepsilon_{1}$ sea neutral sobre el producto de largo plazo, es necesario que se satisfaga la siguiente condición.

$$
\sum_{\mathrm{k}=0}^{\infty} \mathrm{C}_{11}(\mathrm{k})=0
$$


Es decir, los efectos del primer tipo de perturbación sobre el producto se anularán en el largo plazo. Si se conocen las perturbaciones $\left(\varepsilon_{1}\right)$ y los mecanismos de transmisión estructurales de la inflación $\left(\mathrm{C}_{21}(\mathrm{k}), \mathrm{C}_{22}(\mathrm{k}) \forall \mathrm{k}\right)$ es posible descomponer la serie de inflación en dos componentes.

$$
\Delta \Pi=\sum_{\mathrm{k}=0}^{\infty} \mathrm{C}_{21}(\mathrm{k}) \varepsilon_{1 \mathrm{t}-\mathrm{k}}+\sum_{\mathrm{k}=0}^{\infty} \mathrm{C}_{22}(\mathrm{k}) \varepsilon_{2 \mathrm{t}-\mathrm{k}}
$$

donde,

$\sum_{\mathrm{k}=0}^{\infty} \mathrm{C}_{21}(\mathrm{k}) \varepsilon_{1 \mathrm{t}-\mathrm{k}}$ es el componente que define los cambios en la inflación latente.

Sin embargo, como no se conocen los choques estructurales, no es posible estimar directamente los parámetros de la matriz $C(L)$. La metodología Blanchard y Quah permite recobrar la representación estructural, para lo cual se procede de la siguiente manera:

Se estima la forma reducida de un vector autorregresivo (VAR) para estas variables, de manera que cada variable se explica por su propios rezagos y los de la otra variable.

$$
\mathrm{X}_{\mathrm{t}}=\mathrm{A}(\mathrm{L}) \mathrm{X}_{\mathrm{t}-1}+\mathrm{e}_{\mathrm{t}}
$$

Los residuales de esta estimación $\left(\mathrm{e}_{\mathrm{t}}\right)$ contienen la información sobre las perturbaciones $\left(\varepsilon_{1}\right)$ que afectan contemporáneamente al producto y a la inflación en cada periodo. Si esto es cierto para un periodo, también lo es para todos los periodos anteriores, por lo que en principio, el vector $\mathrm{X}_{\mathrm{t}}$ puede representarse como una distribución de rezagos de orden infinito de los residuales. Esta transformación es conocida como transformación de Wold. ${ }^{41}$ La representación en promedios móviles de los residuales sería:

$$
\mathrm{X}_{\mathrm{t}}=\mathrm{D}(\mathrm{L}) \mathrm{e}_{\mathrm{t}} \quad \text { con } \quad \mathrm{ee}^{\prime}=\operatorname{Var}(\mathrm{e})=\Omega
$$

Como e e contiene la información de las perturbaciones estructurales contemporáneas, estas últimas se pueden obtener como una combinación lineal de los residuales. Es decir:

$$
\mathrm{e}_{\mathrm{t}}=\mathrm{C}(0) \varepsilon_{\mathrm{t}}
$$

\footnotetext{
${ }^{41}$ Según el teorema de representación de Wold, bajo condiciones débiles de regularidad cualquier proceso autorregresivo estacionario puede ser representado como un proceso de promedios móviles (MA) invertible de orden infinito. Para la transformación, partiendo de la ecuación (4), se tiene que:

$\mathrm{X}_{\mathrm{t}}-\mathrm{LA}(\mathrm{L}) \mathrm{X}_{\mathrm{t}}=\mathrm{e}_{\mathrm{t}}$

$X_{t}=[I-L A(L)]^{-1} e_{t}$

Sea $D(L)=[I-L A(L)]^{-1}$

Entonces

$\mathrm{X}_{\mathrm{t}}=\mathrm{D}(\mathrm{L}) \mathrm{e}_{\mathrm{t}}$

Para estimar estos coeficientes

$\mathrm{D}(0)=\mathrm{I}$

$D(k)=\sum_{j=1}^{k} A(j) D(k-j)$
} 
Esta combinación lineal debe mantenerse para todos los periodos, por lo que se cumple que $\mathrm{D}(\mathrm{k}) \mathrm{e}_{\mathrm{t}}=\mathrm{C}(\mathrm{k}) \varepsilon_{\mathrm{t}}{ }^{42} \mathrm{y}$ de esta combinación es posible recuperar los parámetros estructurales de los efectos rezagados de las perturbaciones.

$$
\mathrm{C}(\mathrm{k})=\mathrm{D}(\mathrm{k}) \mathrm{C}(0)
$$

D ado que la estimación del VAR y la transformación de Wold permiten conocer los residuales e y las matrices $D(k)$, para recuperar las perturbaciones estructurales $\varepsilon_{\mathrm{t}} \mathrm{y}$ las matrices $C(k)$ mediante las ecuaciones (6) y (7), únicamente se requiere conocer los parámetros de la matriz $C(0)$. Blanchard y Quah proponen un criterio para determinar estos parámetros. Como se requiere estimar cuatro parámetros, se requieren de cuatro restricciones, tres restricciones provienen de supuestos estadísticos y la cuarta de la definición misma de la inflación latente.

D e la matriz de varianza-covarianza de los residuales del VAR y de la ecuación (6) se tiene:

$$
\mathrm{ee}^{\prime}=\Omega=\mathrm{C}(0) \varepsilon \varepsilon^{\prime} \mathrm{C}(0)^{\prime}
$$

Si se supone que las dos perturbaciones estructurales son ortogonales (no correlacionadas) y se normalizan para que su varianza sea unitaria, la matriz de varianza covarianza de las perturbaciones será la identidad y por lo tanto, la ecuación (8) se reduce a:

es decir

$$
\mathrm{ee}^{\prime}=\Omega=\mathrm{C}(0) \mathrm{C}(0)^{\prime}
$$

$$
\left[\begin{array}{cc}
\sigma_{1}^{2} & \sigma_{1,2} \\
\sigma_{1,2} & \sigma_{2}^{2}
\end{array}\right]=\left[\begin{array}{cc}
C_{11}(0)^{2}+C_{12}(0)^{2} & C_{11}(0) C_{21}(0)+C_{12}(0) C_{22}(0) \\
C_{11}(0) C_{21}(0)+C_{12}(0) C_{22}(0) & C_{21}(0)^{2}+C_{22}(0)^{2}
\end{array}\right]
$$

Esta ecuación determina las tres restricciones estadísticas. Se requiere de una cuarta restricción, la cual proviene del supuesto teórico de que el primer tipo de perturbaciones no tiene un efecto de largo plazo sobre el producto, de manera que utilizando la ecuación (7), la condición de largo plazo de la ecuación (2) puede ser reescrita como:

$$
\left\{\sum_{\mathrm{k}=0}^{\infty} \mathrm{D}_{11}(\mathrm{k})\right\} \mathrm{C}_{11}(0)+\left\{\sum_{\mathrm{k}=0}^{\infty} \mathrm{D}_{12}(\mathrm{k})\right\} \mathrm{C}_{21}(0)=0
$$

Una vez impuestas estas cuatro restricciones es posible determinar las perturbaciones y los parámetros estructurales para descomponer en base a la ecuación (3) a los cambios en el crecimiento del IPC en sus componentes latente y no-latente.

\section{INFLACIÓN PERMANENTE}

Alvarez y Sebastián (1995) proponen una modificación a la metodología de Quah y Vahey en cuanto a la clasificación de las perturbaciones estructurales. Su propuesta es clasificar las perturbaciones respecto a su efecto sobre el crecimiento de los precios, de manera que en este caso las perturbaciones que afectan al producto y a la inflación serán $\xi_{1}$ y $\xi_{2}$. D onde $\xi_{2}$ agrupa a las perturbaciones cuyo efecto sobre el crecimiento de los precios desaparece en el mediano al largo plazo, es decir, los choques con un efecto transitorio sobre la inflación. En consecuencia, el primer tipo de perturbaciones $\xi_{1}$ tiene un efecto permanente sobre la inflación.

\footnotetext{
${ }^{42}$ Es importante recordar que $\mathrm{D}(0)=I$
} 
Para estimar la inflación permanente el procedimiento es muy similar al expuesto para la inflación latente y prácticamente consiste en modificar la restricción que en la latente proviene de la teoría económica. La ecuación del VAR estructural para la inflación permanente sería:

$$
\mathrm{X}_{\mathrm{t}}=\mathrm{B}(\mathrm{L}) \xi_{\mathrm{t}}
$$

La restricción de que el segundo tipo de perturbaciones no tiene un efecto permanente sobre la inflación estaría dada por:

$$
\sum_{\mathrm{k}=0}^{\infty} \mathrm{B}_{22}(\mathrm{k})=0
$$

En este caso también es posible descomponer a la inflación en dos componentes:

$$
\Delta \Pi=\sum_{\mathrm{k}=0}^{\infty} \mathrm{B}_{21}(\mathrm{k}) \xi_{1 \mathrm{t}-\mathrm{k}}+\sum_{\mathrm{k}=0}^{\infty} \mathrm{B}_{22}(\mathrm{k}) \xi_{2 \mathrm{t}-\mathrm{k}}
$$

donde la inflación permanente está definida por el primer término del lado derecho de la ecuación (3’).

Para identificar estos componentes se utiliza el mismo VAR en forma reducida y las ecuaciones (6), (7) y (9) son las mismas que las utilizadas para la inflación latente (sustituyendo $C(\bullet)$ por $B(\bullet)$ ). El único cambio adicional para identificar los componentes de $B(0)$ es la cuarta restricción la cual estaría dada por:

$$
\left\{\sum_{\mathrm{k}=0}^{\infty} \mathrm{D}_{21}(\mathrm{k})\right\} \mathrm{B}_{12}(0)+\left\{\sum_{\mathrm{k}=0}^{\infty} \mathrm{D}_{22}(\mathrm{k})\right\} \mathrm{B}_{22}(0)=0
$$




\section{BIBLIOGRAFIA}

Álvarez Luis J. y Miguel Sebastián (1995), “La Inflación Latente en España: Una perspectiva Macroeconómica”, DoumetodeTrabajo9521, Servicio de Estudios, Banco de España.

Álvarez Luis J. y Ma. De los Llanos Matea (1997), "Medidas del Proceso Inflacionista", en La Pdítica Mondariayla InflacoónenEspaña, Servicio de Estudios, Banco de España, pp. 341-376.

Armknecht, Paul A. (1996), "Improving the Efficiency of the U.S. CPI," IMF Wakking Paper WP/ 96/103, September.

Ball, Laurence and Gregory Mankiw (1995), "Relative-Price Changes As Aggregate Supply Shocks," QuartelyJarmal of Economics, 161-193.

Banco de España (1997), "La Pdítica Montaria y la Inflaaón en España”, Servicio de Estudios, Alianza Editorial, Madrid. 863 págs.

Banco de la Reserva del Perú (1998), "La Inflación Subyacente en el Perú", Nđas deEstudios, No. 5, Abril, Gerencia de Estudios Económicos.

Blanchard, Oliver J. y Danny Quah (1989), "The Dynamic Effects of Aggregate Demand and Supply Disturbances." Ameican EconamicReiew, September, vol.79, pp. 655-673.

Bollerslev, Tim (1986), "Generalized Autoregressive Conditional Heteroscedaticity." Journal of Econometrics 31, 307-327.

Bruno, Michael y William Easterly (1995), "Inflation Crises and Long-Run Growth," Wodd Bank Pdigy Rerch WokkingPaper No 1517, Washington:World Bank, September.

Bryan, Michael F. y Stephen G. Cecchetti (1993), "Measuring Core Inflation," N.B.E.R. Working Paper No 4303, March.

Bryan, Michael F., Stephen G. Cecchetti and Rodney L. Wiggins II (1997), "Efficient Inflation Estimation," N.B.E.R. WokkingPaper Na 6183, September.

De Gregorio, José (1992), "The Effects of Inflation on Economic Growth-Lessons from Latin America," EurgpeennEconomic Reiew Vol. 36 (A pril), pp. 417-25.

(1993), "Inflation, Taxation and Long-Run Growth," Jauml of Monłary Economics Vol. 31 (June), pp. 271-98.

Engle, Robert (1982), "Autoregressive Conditional Heteroscedastiity with Estimates of the Variance of United Kingdom Inflation." Econamerica 50 (July), 987-1007.

Espasa, Antoni, et al (1987), "La Inflación Subyacente en la Economía Española: Estimación y Metodología," BdđánEconómice Banco de España, Marzo.

Espasa, Antoni, y J.R. Cancelo (1993), "Caracterización de los Aspectos Esenciales de un Fenómeno Económico Mediante Técnicas Estadísticas de Extracción de Señales", en Espasa A. y 
J.R. Cancelo eds. Méodos Cuantitativos para \& Análisis dela Coyuntura Económica Alianza Editorial, págs. 255-323.

Fischer, Stanley (1993), "The Role of Macroeconomic Faxtors in Growth," Journal of Monetary Economics, Vol. 32 (D ecember), pp. 485-512.

Furest, Rosario y Daniel Vaz (1997), "Hacia una Medición de la Inflación Subyacente en el Uruguay," Reista deEconamáa, Segunda Epoca Vol.1 No.1, Banco Central de Uruguay, pp. 213244.

Gamboa, Rafael (1997), "Efecto de los Precios Administrados Sobre la Inflación." SerieDoamentos de Investigacón D ocumento No. 9709, Banco de México

Hamilton, James D. (1994), “TimeSeriesAnalysis”, Princeton University Press.

Hoover, Kevin (1991), "The Casual D irection between Money and Prices," Jaumal of Montay Econamis 27 (June): 381-423.

L'Hotellenie-Fallois, Pilar (1997), “El Análisis de la Inflación desde la Óptica de la Política Monetaria," en La Pdítica Montania y la Inflacoón en España, Servicio de Estudios, Banco de España, pp. 299-340.

Maravall, A (1989), “La Extracción de Señales y Análisis de Coyuntura,” Revista Españda deEcomamá, 6, 1-2, págs. 109-132.

Matea, Ma. de los Llanos y A.V. Regil (1994), "Métodos para la Extracción de Señales y para la Trimestralización". DoumentodeTrabajo9415. Servicio de Estudios, Banco de España.

Mateos, Calixto y Alejandro Gaytán (1997), "Inflación Latente e Inflación Permanente para México", D ocumento Interno del Banco de México.

Mateos, Calixto y Moisés J. Schwartz (1997), "Metas de Inflación como Instrumento de Política Monetaria”, SerieDoumentos deInvestigacón D ocumento No. 9702, Banco de México.

Pérez López, Alejandro (1998), "La Importancia de los Agregados Monetarios Amplios en la Explicacion del Proceso Inflacionario", Banco de México, mimeo.

Pindyck, R.S. y A. Solimano (1993), "Economic Instability and Aggregate Investment", NBER WorkingPaper no 4830, June.

Quah, Danny and Shaun P. Vahey (1995), "Measuring Core Inflation," The Ecomamic Jaumal, September, 1130-1143.

Sánchez, Mercedes (1998), “Medidas Altemativas e Indicadores de Inflación para Méxiø”, Tesis de Licenciatura, ITAM. México.

Sbordone, Argia, y Kenneth Kuttner (1994), “Does Inflation Reduce Productivity?” Ecomamic Pespetives Vol. 18 (November-D ecember), pp. 2-14.

Smyth, David J., (1994), “Inflation and G rowth,” Jaumal of Macroeconmis Vol. 16 (Spring), pp.261-70. 
Shiratsuka, Shigenori (1997), "Inflation Measures for Monetary Policy: Measuring Underlying Inflation Trend and Its Implications for Monetary Policy Implementation," I.ME.S. Disassion PaperNo 97-E-7, Bank of Japan, August. 